\title{
SHARP ESTIMATES OF THE ONE-DIMENSIONAL BOUNDARY CONTROL COST FOR PARABOLIC SYSTEMS AND APPLICATION TO THE $N$-DIMENSIONAL BOUNDARY NULL CONTROLLABILITY IN CYLINDRICAL DOMAINS*
}

\author{
ASSIA BENABDALLAH ${ }^{\dagger}$, FRANCK BOYER $^{\dagger}$, MANUEL GONZÁLEZ-BURGOS $^{\ddagger}$, AND \\ GUILLAUME OLIVE ${ }^{\dagger}$
}

\begin{abstract}
In this paper we consider the boundary null controllability of a system of $n$ parabolic equations on domains of the form $\Omega=(0, \pi) \times \Omega_{2}$ with $\Omega_{2}$ a smooth domain of $\mathbb{R}^{N-1}, N>1$. When the control is exerted on $\{0\} \times \omega_{2}$ with $\omega_{2} \subset \Omega_{2}$, we obtain a necessary and sufficient condition that completely characterizes the null controllability. This result is obtained through the LebeauRobbiano strategy and requires an upper bound of the cost of the one-dimensional boundary null control on $(0, \pi)$. The latter is obtained using the moment method and it is shown to be bounded by $C e^{C / T}$ when $T$ goes to $0^{+}$.
\end{abstract}

Key words. parabolic systems, boundary controllability, biorthogonal families, Kalman rank condition

AMS subject classifications. 93B05, 93C05, 35K05

DOI. $10.1137 / 130929680$

1. Introduction. The controllability of systems of $n$ partial differential equations by $m<n$ controls is a relatively recent subject. We can quote [LZ98], [dT00], [BN02] among the first works. More recently in [AKBDGB09b], the authors have generalized the so-called Kalman rank condition using fine tools of partial differential equations. This condition usually characterizes the controllability of linear ordinary differential systems (see [KFA69]). In the above reference, the authors prove that a suitable generalized Kalman rank condition provides the distributed null-controllability property of some classes of linear parabolic systems. See also [AKBDGB09a] where the authors provide a Kalman rank condition in the framework of time-dependent coupled linear parabolic systems. On the other hand, while for scalar problems the boundary controllability is known to be equivalent to the distributed controllability, it has been proved in [FCGBdT10] that this is no longer the case for systems. This reveals that the controllability of systems is much more subtle. In [AKBGBdT13a, AKBGBdT13b], it is even shown that a minimal time of control can appear if the diffusion is different on each equation. This is quite surprising for a system possessing an infinite speed of propagation. It is important to emphasize that the previous quoted results concerning the boundary controllability were established in space dimension one. This restriction is mainly due to the fact that they used the moment method, generalizing the works of [FR71, FR75] on the boundary controllability of the one-dimensional scalar heat equation.

In higher space dimensions the boundary controllability of parabolic systems remains widely open and it is the main purpose of this article to give some partial

${ }^{*}$ Received by the editors July 18, 2013; accepted for publication (in revised form) July 9, 2014; published electronically September 25, 2014.

http://www.siam.org/journals/sicon/52-5/92968.html

†Aix Marseille Université, CNRS, Centrale Marseille, 12M, UMR 7373, 13453 Marseille, France (assia.benabdallah@univ-amu.fr, franck.boyer@univ-amu.fr, guillaume.olive@univ-amu.fr).

${ }^{\ddagger}$ Dpto. E.D.A.N. and I.M.U.S., Universidad de Sevilla, Aptdo. 1160, 41080 Sevilla, Spain (manoloburgos@us.es). This author's research was supported by grant MTM2010-15592 of the Ministry of Science and Innovation.

2970 
answers. To our knowledge, the only results on this issue are the one of [ABL12] and [AB12]. In these articles, the results for parabolic systems are deduced from the study of the boundary control problem of two coupled wave equations using transmutation techniques. As a result they rely on some geometric constraints on the control domain. We will see here that those assumptions are not necessary.

Let us also mention [Oli13] for related questions for the approximate controllability problem. We refer to [AKBGBdT11b] for a more detailed survey on the controllability of parabolic systems.

In the present work, we focus on the boundary null controllability of the following $n$ coupled parabolic equations by $m$ controls in dimension $N>1$ :

$$
\left\{\begin{aligned}
\partial_{t} y & =\Delta y+A y & & \text { in }(0, T) \times \Omega, \\
y & =1_{\gamma} B v & & \text { on }(0, T) \times \partial \Omega, \\
y(0) & =y_{0} & & \text { in } \Omega,
\end{aligned}\right.
$$

in the case where the domain $\Omega$ has a Cartesian product structure

$$
\Omega=\Omega_{1} \times \Omega_{2},
$$

where $\Omega_{i} \subset \mathbb{R}^{N_{i}}, i=1,2$, are bounded open regular domains. In (1.1), $T>0$ is the control time, the nonempty relative subset $\gamma \subset \partial \Omega$ is the control domain, $y$ is the state, $y_{0}$ is the initial datum, $A \in \mathcal{M}_{n}(\mathbb{C})$ and $B \in \mathcal{M}_{n \times m}(\mathbb{C})$ are constant matrices, and $v$ is the boundary control.

Under appropriate assumptions we show that the controllability of system (1.1) is reduced to the controllability of the same system posed in $\Omega_{1}$ (see Theorem 1.3 below). The proof is based on the method of Lebeau-Robiano [LR95]. This strategy (already used in a different framework in [BDR07]) requires an estimate of the cost of the $N_{1}$-dimensional control with respect to the control time when $T \rightarrow 0^{+}$.

In a second part, we establish that the cost of the one-dimensional null control on $(0, T)$ is bounded by $C e^{C / T}$ for some $C>0$, as $T \rightarrow 0^{+}$(see Theorem 1.4 below). This is the second main result of this paper. It shows in particular that our first result above can be applied at least in the case $N_{1}=1$. Observe that the results obtained in [AKBGBdT11a] do not permit to deduce the required exponential estimate on the null-control cost. The demonstration of this result follows the approach of [FR71] and [Mil04] (for the scalar case). In the scalar case, [Sei84] (see also [FCZ00]) gave a similar estimate of the cost of the boundary control of the heat equation, which is known to be optimal thanks to the work [Güi85].

Note finally, that the extension of the present results to more general domains $\Omega$ in $\mathbb{R}^{N}$ as well as the study of the case with a different diffusion coefficient in each equation, remains an open problem.

1.1. Reminders and notation. Let us first recall that system (1.1) is wellposed in the sense that, for every $y_{0} \in H^{-1}(\Omega)^{n}$ and $v \in L^{2}\left(0, T ; L^{2}(\partial \Omega)^{m}\right)$, there exists a unique solution $y \in C^{0}\left([0, T] ; H^{-1}(\Omega)^{n}\right) \cap L^{2}\left(0, T ; L^{2}(\Omega)^{n}\right)$, defined by transposition. Moreover, this solution depends continuously on the initial datum $y_{0}$ and the control $v$. More precisely,

$$
\|y\|_{C^{0}\left([0, T] ; H^{-1}(\Omega)^{n}\right)} \leq C e^{C T}\left(\left\|y_{0}\right\|_{H^{-1}(\Omega)^{n}}+\|v\|_{L^{2}\left(0, T ; L^{2}(\partial \Omega)^{m}\right)}\right),
$$

where here and all during this work $C>0$ denotes a generic positive constant that may change line to line but which does not depend on $T$ or $y_{0}$. We shall also sometimes use the notation $C^{\prime}, C^{\prime \prime}$, and so on. 
Let us now make precise the concept of controllability which we will deal with in this paper. We say that system (1.1) is null controllable at time $T$ if for every $y_{0} \in$ $H^{-1}(\Omega)^{n}$, there exists a control $v \in L^{2}\left(0, T ; L^{2}(\partial \Omega)^{m}\right)$ such that the corresponding solution $y$ satisfies

$$
y(T)=0
$$

In such a case, it is well known that there exists $C_{T}>0$ such that

$$
\|v\|_{L^{2}\left(0, T ; L^{2}(\partial \Omega)^{m}\right)} \leq C_{T}\left\|y_{0}\right\|_{H^{-1}(\Omega)^{n}} \quad \forall y_{0} \in H^{-1}(\Omega)^{n} .
$$

The infimum of the constants $C_{T}$ satisfying (1.3) is called the cost of the null control at time $T$.

Remark 1. Replacing $y(t)$ by $e^{-\mu t} y(t)$ and $A$ by $A-\mu$, with $\mu>0$, we can assume without loss of generality that the matrix $A$ is stable, i.e., that all its eigenvalues have a negative real part.

Finally, let us recall the well-known duality between controllability and observability (for the general theory which relates observability and controllability, see [DR77]).

THEOREM 1.1. Let $E$ be a closed subspace of $H_{0}^{1}(\Omega)^{n}$ and set $E^{-1}=-\Delta E \subset$ $H^{-1}(\Omega)^{n}$. Let us denote by $\Pi_{E}$ (resp., $\Pi_{E^{-1}}$ ) the orthogonal projection on $E$ (resp., $\left.E^{-1}\right)$. Let $C_{T}>0$ be fixed. For every $y_{0} \in E^{-1}$ there exists a control

$$
v \in L^{2}\left(0, T ; L^{2}(\partial \Omega)^{m}\right)
$$

such that

$$
\left\{\begin{array}{l}
\Pi_{E^{-1}} y(T)=0, \\
\|v\|_{L^{2}\left(0, T ; L^{2}(\partial \Omega)^{m}\right)} \leq C_{T}\left\|y_{0}\right\|_{H^{-1}(\Omega)^{n}},
\end{array}\right.
$$

where $y$ is the corresponding solution to (1.1), if and only if

$$
\left\|\Pi_{E} z(0)\right\|_{H_{0}^{1}(\Omega)^{n}}^{2} \leq C_{T}^{2} \int_{0}^{T}\left\|1_{\gamma} B^{*} \partial_{n} z(t)\right\|_{L^{2}(\partial \Omega)^{m}}^{2} d t \quad \forall z_{T} \in E,
$$

where $z$ is the solution to the adjoint system

$$
\left\{\begin{aligned}
-\partial_{t} z & =\Delta z+A^{*} z & & \text { in }(0, T) \times \Omega, \\
z & =0 & & \text { on }(0, T) \times \partial \Omega, \\
z(0) & =z_{T} & & \text { in } \Omega .
\end{aligned}\right.
$$

Notation. We gather here some standard notation that we shall use throughout this paper. For any real numbers $a<b$ we denote $\llbracket a, b \rrbracket=[a, b] \cap \mathbb{Z}$. For $z \in \mathbb{C}, \Re(z)$ and $\Im(z)$ denote the real and imaginary parts of $z$. Finally, $x \in \mathbb{R} \mapsto\lfloor x\rfloor \in \mathbb{Z}$ denotes the floor function.

\subsection{Main results.}

1.2.1. Boundary controllability for a multidimensional parabolic system. The first main achievement of this work is the following.

TheOrem 1.2. Let $\omega_{2} \subset \Omega_{2}$ be a nonempty open subset and take $\Omega_{1}=(0, \pi)$. Then, system (1.1) is null controllable at time $T$ on $\gamma=\{0\} \times \omega_{2}$ if and only if

$$
\operatorname{rank}\left(B_{k}\left|A_{k} B_{k}\right| A_{k}^{2} B_{k}|\cdots| A_{k}^{n k-1} B_{k}\right)=n k \quad \forall k \geq 1,
$$

Copyright $@$ by SIAM. Unauthorized reproduction of this article is prohibited. 


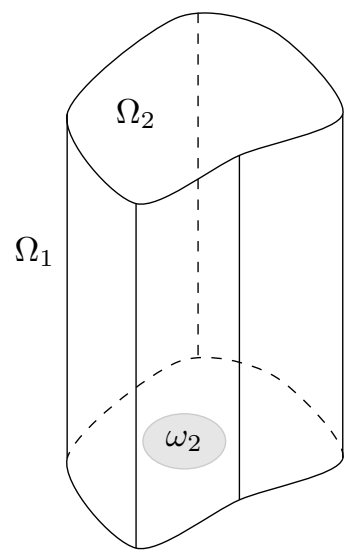

FIG. 1. Typical geometric situation.

where we have introduced the following notation (recall that $\lambda_{j}=j^{2}$ ):

$$
\begin{aligned}
A_{k} & =\left(\begin{array}{ccccc}
-\lambda_{1}+A & 0 & \cdots & \cdots & 0 \\
0 & -\lambda_{2}+A & \ddots & & \vdots \\
\vdots & \ddots & \ddots & \ddots & \vdots \\
\vdots & & \ddots & \ddots & 0 \\
0 & \cdots & \cdots & 0 & -\lambda_{k}+A
\end{array}\right) \in \mathcal{M}_{n k}(\mathbb{C}), \\
B_{k} & =\left(\begin{array}{c}
B \\
B \\
\vdots \\
\vdots \\
B
\end{array}\right) \in \mathcal{M}_{n k \times m}(\mathbb{C}) .
\end{aligned}
$$

One may think of a cylindrical domain where the control domain is a subset of the top or bottom face (see Figure 1).

This result will be obtained as a corollary of some other theorems that are important results too. The first one is the following and should be connected with [Fat75] and [Mil05].

TheOREM 1.3. Let $\gamma_{1} \subset \partial \Omega_{1}$ be a nonempty relative subset. Assume that the following $N_{1}$-dimensional system

$$
\left\{\begin{aligned}
\partial_{t} y^{1} & =\Delta_{x_{1}} y^{1}+A y^{1} & & \text { in }(0, T) \times \Omega_{1}, \\
y^{1} & =1_{\gamma_{1}} B v^{1} & & \text { on }(0, T) \times \partial \Omega_{1}, \\
y^{1}(0) & =y_{0}^{1} & & \text { in } \Omega_{1},
\end{aligned}\right.
$$

is null controllable for any time $T>0$ with, in addition, the following bound for the control $\operatorname{cost} C_{T}^{\Omega_{1}}$ :

$$
C_{T}^{\Omega_{1}} \leq C e^{C / T} \quad \forall T>0
$$

Copyright $@$ by SIAM. Unauthorized reproduction of this article is prohibited. 
Then, for any nonempty open set $\omega_{2} \subset \Omega_{2}$ the $N$-dimensional system (1.1) is null controllable at any time $T>0$ on the control domain $\gamma=\gamma_{1} \times \omega_{2}$.

Remark 2. The converse of Theorem 1.3 also holds. More precisely, if the $N$ dimensional system (1.1) is null controllable at time $T$, then the $N_{1}$-dimensional system (1.7) is also null controllable at time $T$. This can be proved using a Fourier decomposition in the direction of $\Omega_{2}$.

It is worth mentioning that such a decomposition also shows that, when $\omega_{2}=\Omega_{2}$, the proof of Theorem 1.3 is much simpler and it does not need the control cost estimate (1.8). Moreover, the domain $\Omega_{2}$ can even be unbounded in this case.

1.2.2. Estimate of the control cost for a one-dimensional boundary controllability problem. The third result of this paper provides an important example where Theorem 1.3 can be successfully applied.

More precisely, we show that the assumption (1.8) on the short time behavior of the control cost actually holds in the one-dimensional case for the following system if we assume the rank condition (1.5)

$$
\begin{cases}\partial_{t} y=\partial_{x x}^{2} y+A y & \text { in }(0, T) \times(0, \pi), \\ y(t, 0)=B v(t), y(t, \pi)=0 & \text { in }(0, T), \\ y(0)=y_{0} & \text { in }(0, \pi) .\end{cases}
$$

We recall that it has been established in [AKBGBdT11a] that system (1.9) is null controllable at time $T>0$ if and only if the rank condition (1.5) holds.

However, in the abovementioned reference, no estimate on the control cost is provided. This is the next goal of the present paper: to give a more precise insight into the proof of the controllability result for system (1.9) that allows a precise estimate of the control cost as a function of $T$.

TheOREM 1.4. Assume that the rank condition (1.5) holds. Then, for every $T>0$ and $y_{0} \in H^{-1}(0, \pi)^{n}$ there exists a null control $v \in L^{2}(0, T)^{m}$ for system (1.9) which, in addition, satisfies

$$
\|v\|_{L^{2}(0, T)^{m}} \leq C e^{C / T}\left\|y_{0}\right\|_{H^{-1}(0, \pi)^{n}} .
$$

This theorem, combined with Theorem 1.3 and Remark 2, gives a proof of Theorem 1.2.

1.2.3. Bounds on biorthogonal families of exponentials. The proof of Theorem 1.4 is mainly based on the existence of a suitable biorthogonal family of timedependent exponential functions. The construction provided in [AKBGBdT11a] does not allow us to estimate the control cost. That is the reason why we propose here a slightly different approach which is the key to obtaining the factor $e^{C / T}$. This abstract result, which is interesting in itself and potentially useful in other situations, can be formulated as follows.

ThEOREM 1.5. Let $\left\{\Lambda_{k}\right\}_{k \geq 1} \subset \mathbb{C}$ be a sequence of complex numbers fulfilling the following assumptions:

1. $\Lambda_{k} \neq \Lambda_{n}$ for all $k, n \in \mathbb{N}$ with $k \neq n$;

2. $\Re\left(\Lambda_{k}\right)>0$ for every $k \geq 1$;

3. for some $\beta>0$,

$$
\left|\Im\left(\Lambda_{k}\right)\right| \leq \beta \sqrt{\Re\left(\Lambda_{k}\right)} \quad \forall k \geq 1
$$

Copyright (c) by SIAM. Unauthorized reproduction of this article is prohibited. 
4. $\left\{\Lambda_{k}\right\}_{k \geq 1}$ is nondecreasing in modulus,

$$
\left|\Lambda_{k}\right| \leq\left|\Lambda_{k+1}\right| \quad \forall k \geq 1
$$

5. $\left\{\Lambda_{k}\right\}_{k \geq 1}$ satisfies the following gap condition: for some $\rho, q>0$,

$$
\left\{\begin{array}{l}
\left|\Lambda_{k}-\Lambda_{n}\right| \geq \rho\left|k^{2}-n^{2}\right| \quad \forall k, n:|k-n| \geq q, \\
\inf _{k \neq n:|k-n|<q}\left|\Lambda_{k}-\Lambda_{n}\right|>0
\end{array}\right.
$$

6. for some $p, \alpha>0$,

$$
|p \sqrt{r}-\mathcal{N}(r)| \leq \alpha \quad \forall r>0,
$$

where $\mathcal{N}$ is the counting function associated with the sequence $\left\{\Lambda_{k}\right\}_{k \geq 1}$, that is the function defined by

$$
\mathcal{N}(r)=\#\left\{k:\left|\Lambda_{k}\right| \leq r\right\} \quad \forall r>0 .
$$

Then, there exists $T_{0}>0$ such that, for every $\eta \geq 1$ and $0<T<T_{0}$, we can find a family of $\mathbb{C}$-valued functions

$$
\left\{\varphi_{k, j}\right\}_{k \geq 1, j \in \llbracket 0, \eta-1 \rrbracket} \subset L^{2}(-T / 2, T / 2)
$$

biorthogonal $^{1}$ to $\left\{e_{k, j}\right\}_{k \geq 1, j \in \llbracket 0, \eta-1 \rrbracket}$, where for every $t \in(-T / 2, T / 2)$,

$$
e_{k, j}(t)=t^{j} e^{-\Lambda_{k} t}
$$

with, in addition,

$$
\left\|\varphi_{k, j}\right\|_{L^{2}(-T / 2, T / 2)} \leq C e^{C \sqrt{\Re\left(\Lambda_{k}\right)}+\frac{C}{T}}
$$

for any $k \geq 1, j \in \llbracket 0, \eta-1 \rrbracket$.

2. Boundary null controllability on product domains. This section is devoted to the proof of Theorem 1.3.

2.1. Settings and preliminary remarks. Let $\lambda_{j}^{\Omega_{1}}$ (resp., $\left.\lambda_{j}^{\Omega_{2}}\right), j \geq 1$, be the Dirichlet eigenvalues of the Laplacian on $\Omega_{1}$ (resp., $\Omega_{2}$ ), and let $\phi_{j}^{\Omega_{1}}$ (resp., $\phi_{j}^{\Omega_{2}}$ ) be the corresponding normalized eigenfunctions.

Let us introduce the (closed) subspaces of $H_{0}^{1}(\Omega)^{n}$ on which we will establish the partial observability later on (section 2.2):

$$
E_{J}=\left\{\sum_{j=1}^{J}\left\langle u, \phi_{j}^{\Omega_{2}}\right\rangle_{L^{2}\left(\Omega_{2}\right)} \phi_{j}^{\Omega_{2}} \mid u \in H_{0}^{1}(\Omega)^{n}\right\} \subset H_{0}^{1}(\Omega)^{n}, \quad J \geq 1,
$$

where the notation $\sum_{j=1}^{J}\left\langle u, \phi_{j}^{\Omega_{2}}\right\rangle_{L^{2}\left(\Omega_{2}\right)} \phi_{j}^{\Omega_{2}}$ is used to mean the function

$$
\left(x_{1}, x_{2}\right) \in \Omega \longmapsto \sum_{j=1}^{J}\left\langle u\left(x_{1}, \cdot\right), \phi_{j}^{\Omega_{2}}\right\rangle_{L^{2}\left(\Omega_{2}\right)} \phi_{j}^{\Omega_{2}}\left(x_{2}\right) .
$$

\footnotetext{
${ }^{1}$ That is $\left\langle\varphi_{k, j}, e_{l, \nu}\right\rangle_{L^{2}(-T / 2, T / 2)}=\int_{-T / 2}^{T / 2} \varphi_{k, j}(t) \overline{e_{l, \nu}}(t) d t=\delta_{k l} \delta_{j \nu}$.
}

Copyright $@$ by SIAM. Unauthorized reproduction of this article is prohibited. 
We then define the "dual" spaces of $E_{J}$

$$
E_{J}^{-1}=-\Delta E_{J} \subset H^{-1}(\Omega)^{n}, \quad J \geq 1 .
$$

Let us recall that we denote by $\Pi_{E_{J}}$ (resp., $\Pi_{E_{J}^{-1}}$ ) the orthogonal projection in $H_{0}^{1}(\Omega)^{n}$ (resp., $H^{-1}(\Omega)^{n}$ ) onto $E_{J}$ (resp., $E_{J}^{-1}$ ). It is not difficult to see that we have the relation $\Pi_{E_{J}^{-1}}(-\Delta u)=-\Delta \Pi_{E_{J}} u$ for any $u \in H_{0}^{1}(\Omega)^{n}$.

Lemma 2.1. For any $u \in H_{0}^{1}(\Omega)^{n}$ we have

$$
u=\sum_{j=1}^{+\infty}\left\langle u, \phi_{j}^{\Omega_{2}}\right\rangle_{L^{2}\left(\Omega_{2}\right)} \phi_{j}^{\Omega_{2}} .
$$

It follows from this lemma that $\Pi_{E_{J}} u=\sum_{j=1}^{J}\left\langle u, \phi_{j}^{\Omega_{2}}\right\rangle_{L^{2}\left(\Omega_{2}\right)} \phi_{j}^{\Omega_{2}}$ for any $u \in$ $H_{0}^{1}(\Omega)^{n}$.

Proof of Lemma 2.1. Let us show that the sequence $\left\{S_{J} u\right\}_{J \geq 1}$ defined by

$$
S_{J} u=\sum_{j=1}^{J}\left\langle u, \phi_{j}^{\Omega_{2}}\right\rangle_{L^{2}\left(\Omega_{2}\right)} \phi_{j}^{\Omega_{2}}
$$

is a Cauchy sequence of $H_{0}^{1}(\Omega)^{n}$. For any $J>K \geq 1$ we have

$$
\begin{aligned}
& \left\|S_{J} u-S_{K} u\right\|_{H_{0}^{1}(\Omega)^{n}}^{2} \\
& =\left\|\sum_{j=K+1}^{J}\left\langle u, \phi_{j}^{\Omega_{2}}\right\rangle_{L^{2}\left(\Omega_{2}\right)} \phi_{j}^{\Omega_{2}}\right\|_{H_{0}^{1}(\Omega)^{n}}^{2}\left\|_{j=K+1}^{J}\right\|\left\langle u, \phi_{j}^{\Omega_{2}}\right\rangle_{L^{2}\left(\Omega_{2}\right)}\left\|_{H_{0}^{1}\left(\Omega_{1}\right)^{n}}^{2}+\sum_{j=K+1}^{\Omega_{j}}\right\|\left\langle u, \phi_{j}^{\Omega_{2}}\right\rangle_{L^{2}\left(\Omega_{2}\right)} \|_{L^{2}\left(\Omega_{1}\right)^{n}}^{2} .
\end{aligned}
$$

Using Lebesgue's dominated convergence theorem it is not difficult to see that these terms go to zero as $J, K \rightarrow+\infty$. As a result $S_{J} u \frac{H_{0}^{1}}{J \rightarrow+\infty} v$ for some $v \in H_{0}^{1}(\Omega)^{n}$. In particular, $\left\langle v, \phi_{k}^{\Omega_{1}} \phi_{j}^{\Omega_{2}}\right\rangle_{L^{2}(\Omega)}=\left\langle u, \phi_{k}^{\Omega_{1}} \phi_{j}^{\Omega_{2}}\right\rangle_{L^{2}(\Omega)}$ for every $j, k \geq 1$, and it follows that $v=u$.

2.2. Partial observability. One of the key points in making use of the LebeauRobbiano strategy is the estimate of the cost of the partial observabilities on the approximation subspaces. This will be used for the active control phase.

Proposition 2.2. Let $\Omega_{2}$ be of class $C^{2}$. Assume that system (1.7) is controllable at time $T$ with $\operatorname{cost} C_{T}^{\Omega_{1}}$. Then,

$$
\left\|\Pi_{E_{J}} z(0)\right\|_{H_{0}^{1}(\Omega)^{n}}^{2} \leq C\left(C_{T}^{\Omega_{1}}\right)^{2} e^{C \sqrt{\lambda_{J}^{\Omega_{2}}}} \int_{0}^{T}\left\|1_{\gamma_{1} \times \omega_{2}} B^{*} \partial_{n} z(t)\right\|_{L^{2}(\partial \Omega)^{m}}^{2} d t \quad \forall z_{T} \in E_{J},
$$

where $z$ is the solution to the adjoint system (1.4).

By Theorem 1.1 we deduce the following.

Corollary 2.3. For every $J \geq 1$ and $y_{0} \in E_{J}^{-1}$ there exists a control $v\left(y_{0}\right) \in$ $L^{2}\left(0, T ; L^{2}(\partial \Omega)^{m}\right)$ with

$$
\left\|v\left(y_{0}\right)\right\|_{L^{2}\left(0, T ; L^{2}(\partial \Omega)^{m}\right)} \leq C\left(C_{T}^{\Omega_{1}}\right) e^{C \sqrt{\lambda_{J}^{\Omega_{2}}}}\left\|y_{0}\right\|_{H^{-1}(\Omega)^{n}},
$$

Copyright $@$ by SIAM. Unauthorized reproduction of this article is prohibited. 
such that the solution y to system (1.1) satisfies

$$
\Pi_{E_{J}^{-1}} y(T)=0 .
$$

Proof of Proposition 2.2. Let $z_{T} \in E_{J}$ so that

$$
z_{T}\left(x_{1}, x_{2}\right)=\sum_{j=1}^{J} z_{T}^{j}\left(x_{1}\right) \phi_{j}^{\Omega_{2}}\left(x_{2}\right)
$$

for some $z_{T}^{j} \in H_{0}^{1}\left(\Omega_{1}\right)^{n}$. Let $z$ be the solution of (1.4), the adjoint system of (1.1), associated with $z_{T}$. Thus,

$$
z\left(t, x_{1}, x_{2}\right)=\sum_{j=1}^{J} z^{j}\left(t, x_{1}\right) \phi_{j}^{\Omega_{2}}\left(x_{2}\right),
$$

where $z^{j}$ is the solution to

$$
\left\{\begin{aligned}
-\partial_{t} z^{j} & =\left(\Delta_{x_{1}}-\lambda_{j}^{\Omega_{2}}\right) z^{j}+A^{*} z^{j} & & \text { in }(0, T) \times \Omega_{1}, \\
z^{j} & =0 & & \text { on }(0, T) \times \partial \Omega_{1}, \\
z^{j}(T) & =z_{T}^{j} & & \text { in } \Omega_{1} .
\end{aligned}\right.
$$

Note that $\Pi_{E_{J}} z(0)=z(0)$. A computation of $\|z(0)\|_{H_{0}^{1}(\Omega)^{n}}^{2}$ gives

$$
\|z(0)\|_{H_{0}^{1}(\Omega)^{n}}^{2}=\sum_{j=1}^{J}\left\|z^{j}(0)\right\|_{H_{0}^{1}\left(\Omega_{1}\right)^{n}}^{2}+\sum_{j=1}^{J} \lambda_{j}^{\Omega_{2}}\left\|z^{j}(0)\right\|_{L^{2}\left(\Omega_{1}\right)^{n}}^{2} .
$$

Using the Poincaré inequality we obtain

$$
\|z(0)\|_{H_{0}^{1}(\Omega)^{n}}^{2} \leq C \lambda_{J}^{\Omega_{2}} \sum_{j=1}^{J}\left\|z^{j}(0)\right\|_{H_{0}^{1}\left(\Omega_{1}\right)^{n}}^{2}
$$

Observe now that $z^{j}(t)=e^{-(T-t) \lambda_{j}^{\Omega_{2}}} \psi(t)$, where $\psi$ is the solution to the adjoint system of (1.7) associated with $z_{T}^{j}$. Thus, using the assumption that (1.7) is controllable with $\operatorname{cost} C_{T}^{\Omega_{1}}$, we obtain by Theorem 1.1 that

$$
\left\|z^{j}(0)\right\|_{H_{0}^{1}\left(\Omega_{1}\right)^{n}}^{2} \leq\left(C_{T}^{\Omega_{1}}\right)^{2} \int_{0}^{T}\left\|1_{\gamma_{1}} B^{*} \partial_{n_{1}} z^{j}(t)\right\|_{L^{2}\left(\partial \Omega_{1}\right)^{m}}^{2} d t,
$$

where $n_{1}$ denotes the unit outward normal vector of $\Omega_{1}$. Combined with (2.3), this gives

$$
\|z(0)\|_{H_{0}^{1}(\Omega)^{n}}^{2} \leq C\left(C_{T}^{\Omega_{1}}\right)^{2} \lambda_{J}^{\Omega_{2}} \int_{0}^{T} \sum_{j=1}^{J}\left\|1_{\gamma_{1}} B^{*} \partial_{n_{1}} z^{j}(t)\right\|_{L^{2}\left(\partial \Omega_{1}\right)^{m}}^{2} d t .
$$

Let us denote by $B_{k}$ the $k$ th column of $B$. Applying the Lebeau-Robbiano's spectral inequality [LR95] (see also [LR07, section 3.A $]^{2}$ )

$$
\sum_{j=1}^{J}\left|a_{j}\right|^{2} \leq C e^{C \sqrt{\lambda_{J}^{\Omega_{2}}}} \int_{\omega_{2}}\left|\sum_{j=1}^{J} a_{j} \phi_{j}^{\Omega_{2}}\left(x_{2}\right)\right|^{2} d x_{2}
$$

\footnotetext{
${ }^{2}$ See also [TT11, Theorem 1.5] when $\Omega_{2}$ is a rectangular domain.
}

Copyright $@$ by SIAM. Unauthorized reproduction of this article is prohibited. 
to the sequence of scalars $a_{j}=B_{k}^{*} \partial_{n_{1}} z^{j}\left(t, \sigma_{1}\right), \sigma_{1} \in \partial \Omega_{1}$ being fixed, and summing over $1 \leq k \leq m$, this gives

$$
\sum_{j=1}^{J}\left|B^{*} \partial_{n_{1}} z^{j}\left(t, \sigma_{1}\right)\right|_{\mathbb{C}^{n}}^{2} \leq C e^{C \sqrt{\lambda_{J}^{\Omega_{2}}}} \int_{\omega_{2}}\left|\sum_{j=1}^{J} B^{*} \partial_{n_{1}} z^{j}\left(t, \sigma_{1}\right) \phi_{j}^{\Omega_{2}}\left(x_{2}\right)\right|_{\mathbb{C}^{n}}^{2} d x_{2} .
$$

To conclude it only remains to integrate over $\gamma_{1}$ and to observe that

$$
n(\sigma)=\left(\begin{array}{c}
n_{1}\left(\sigma_{1}\right) \\
0
\end{array}\right) \text { for } \sigma=\left(\sigma_{1}, x_{2}\right) \in \partial \Omega_{1} \times \Omega_{2} .
$$

2.3. Dissipation along the direction $\boldsymbol{\Omega}_{2}$. The other point of the LebeauRobbiano strategy relies on the natural dissipation of the system when no control is exerted (the passive phase). For our purpose, we need an exponential dissipation in the direction $\Omega_{2}$.

PRoposition 2.4. If there is no control on $\left(t_{0}, t_{1}\right)$ (i.e., $v=0$ on $\left(t_{0}, t_{1}\right)$ ) and the corresponding solution $y$ of system (1.1) satisfies

$$
\Pi_{E_{J}^{-1}} y\left(t_{0}\right)=0
$$

then we have the following dissipation estimate

$$
\|y(t)\|_{H^{-1}(\Omega)^{n}} \leq C e^{-\lambda_{J+1}^{\Omega_{2}}\left(t-t_{0}\right)}\left\|y\left(t_{0}\right)\right\|_{H^{-1}(\Omega)^{n}} \quad \forall t \in\left(t_{0}, t_{1}\right) .
$$

Proof. Let $y\left(t_{0}\right)=-\Delta \tilde{y}_{0}, \tilde{y}_{0} \in H_{0}^{1}(\Omega)^{n}$. The assumption $\Pi_{E_{J}^{-1}} y\left(t_{0}\right)=0$ translates into $\Pi_{E_{J}} \tilde{y}_{0}=0$.

Let $\tilde{y}$ be the solution in $H_{0}^{1}(\Omega)^{n}$ to

$$
\left\{\begin{aligned}
\partial_{t} \tilde{y} & =\Delta \tilde{y}+A \tilde{y} & & \text { in }\left(t_{0}, t_{1}\right) \times \Omega \\
\tilde{y} & =0 & & \text { on }\left(t_{0}, t_{1}\right) \times \partial \Omega \\
\tilde{y}\left(t_{0}\right) & =\tilde{y}_{0} & & \text { in } \Omega .
\end{aligned}\right.
$$

Since the matrix $A$ is constant, we can check that

$$
y=-\Delta \tilde{y} \quad \text { in }\left(t_{0}, t_{1}\right) \times \Omega
$$

and thus

$$
\|y(t)\|_{H^{-1}(\Omega)^{n}}=\|\tilde{y}(t)\|_{H_{0}^{1}(\Omega)^{n}}, \quad\left\|y\left(t_{0}\right)\right\|_{H^{-1}(\Omega)^{n}}=\left\|\tilde{y}_{0}\right\|_{H_{0}^{1}(\Omega)^{n}} .
$$

As a consequence it only remains to prove the dissipation for regular data, namely,

$$
\|\tilde{y}(t)\|_{H_{0}^{1}(\Omega)^{n}} \leq C e^{-\lambda_{J+1}^{\Omega_{2}}\left(t-t_{0}\right)}\left\|\tilde{y}_{0}\right\|_{H_{0}^{1}(\Omega)^{n}} \quad \forall t \in\left(t_{0}, t_{1}\right)
$$

for $\tilde{y}_{0}$ such that $\Pi_{E_{J}} \tilde{y}_{0}=0$, i.e., of the form (see Lemma 2.1)

$$
\tilde{y}_{0}=\sum_{j=J+1}^{+\infty} \tilde{y}_{0, j} \phi_{j}^{\Omega_{2}}, \quad \tilde{y}_{0, j}=\left\langle\tilde{y}_{0}, \phi_{j}^{\Omega_{2}}\right\rangle_{L^{2}\left(\Omega_{2}\right)^{n}} \in H_{0}^{1}\left(\Omega_{1}\right)^{n} .
$$

Since $\Pi_{E_{J}} \tilde{y}_{0}=0$ and $A$ is constant, we have $\Pi_{E_{J}} \tilde{y}(t)=0$ for every $t \in\left(t_{0}, t_{1}\right)$ and as a result the following inequalities hold:

$$
\begin{aligned}
\lambda_{J+1}^{\Omega_{2}}\|\tilde{y}(t)\|_{L^{2}(\Omega)^{n}}^{2} & \leq\|\nabla \tilde{y}(t)\|_{L^{2}(\Omega)^{n}}^{2} & \forall t \in\left(t_{0}, t_{1}\right), \\
\lambda_{J+1}^{\Omega_{2}}\|\nabla \tilde{y}(t)\|_{L^{2}(\Omega)^{n}}^{2} & \leq\|\Delta \tilde{y}(t)\|_{L^{2}(\Omega)^{n}}^{2} & \text { for a.e. } t \in\left(t_{0}, t_{1}\right) .
\end{aligned}
$$

Copyright $@$ by SIAM. Unauthorized reproduction of this article is prohibited. 
Using now standard energy estimates and the fact that the matrix $A$ is constant and stable (see Remark 1), we finally obtain the desired dissipation

$$
\|\tilde{y}(t)\|_{H_{0}^{1}(\Omega)^{n}} \leq C e^{-\lambda_{J+1}^{\Omega_{2}}\left(t-t_{0}\right)}\left\|\tilde{y}_{0}\right\|_{H_{0}^{1}(\Omega)^{n}} .
$$

2.4. Lebeau-Robbiano time procedure. We are now ready to prove Theorem 1.3

Let $y_{0} \in H^{-1}(\Omega)^{n}$ be fixed. Let us decompose the interval $[0, T)$ as follows:

$$
[0, T)=\bigcup_{k=0}^{+\infty}\left[a_{k}, a_{k+1}\right]
$$

with

$$
a_{0}=0, \quad a_{k+1}=a_{k}+2 T_{k}, \quad T_{k}=M 2^{-k \rho},
$$

where $\rho \in\left(0, \frac{1}{N_{2}}\right)$ and $M=\frac{T}{2}\left(1-2^{-\rho}\right)$ has been determined to ensure that $2 \sum_{k=0}^{+\infty} T_{k}=$ $T$.

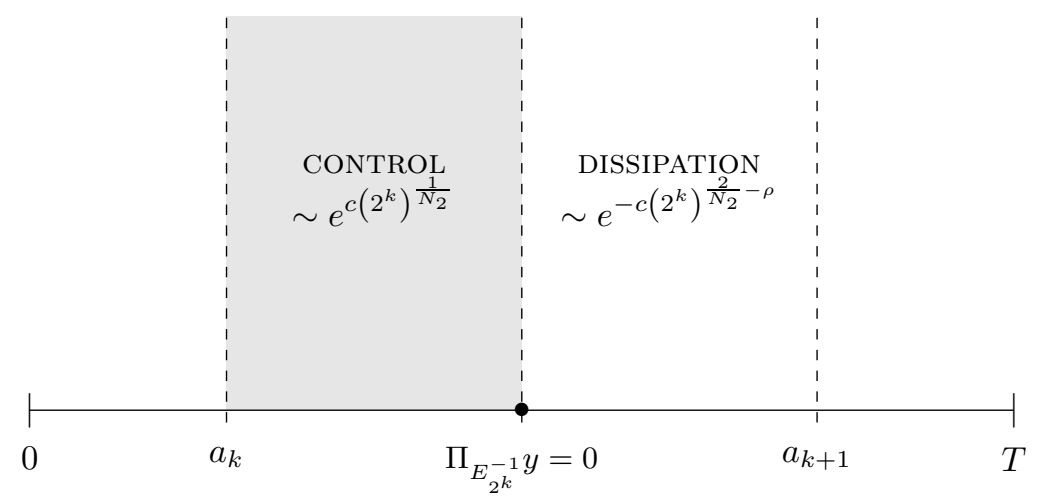

We define the control $v$ and the corresponding solution $y$ piecewise and by induction as follows:

$$
v(t)= \begin{cases}v\left(\Pi_{E_{2^{k}}^{-1}} y\left(a_{k}\right)\right)(t) & \text { if } t \in\left(a_{k}, a_{k}+T_{k}\right), \\ 0 & \text { if } t \in\left(a_{k}+T_{k}, a_{k+1}\right) .\end{cases}
$$

Let us show that $v$ belongs to $L^{2}\left(0, T ; L^{2}(\partial \Omega)^{m}\right)$ and steers $y$ to 0 at time $T$.

Step 1: Estimate on the interval $\left[a_{k}, a_{k}+T_{k}\right]$. From the continuous dependence with respect to the data (1.2) and since $T_{k} \leq T$ we know that

$$
\left\|y\left(a_{k}+T_{k}\right)\right\|_{H^{-1}(\Omega)^{n}} \leq C\left(\left\|y\left(a_{k}\right)\right\|_{H^{-1}(\Omega)^{n}}+\|v\|_{L^{2}\left(a_{k}, a_{k}+T_{k} ; L^{2}(\partial \Omega)^{m}\right)}\right) .
$$

Using the estimate of the cost of the control (2.2) we have

$$
\|v\|_{L^{2}\left(a_{k}, a_{k}+T_{k} ; L^{2}(\partial \Omega)^{m}\right)} \leq C C_{T_{k}}^{\Omega_{1}} e^{C \sqrt{\lambda_{2^{k}}^{\Omega_{2}}}}\left\|\Pi_{E_{2^{k}}^{-1}} y\left(a_{k}\right)\right\|_{H^{-1}(\Omega)^{n}}
$$

and since $\left\|\Pi_{E_{2^{k}}^{-1}}\right\| \mathcal{L}\left(H^{-1}\right) \leq 1$, this gives

$$
\|v\|_{L^{2}\left(a_{k}, a_{k}+T_{k} ; L^{2}(\partial \Omega)^{m}\right)} \leq C C_{T_{k}}^{\Omega_{1}} e^{C \sqrt{\lambda_{2^{k}}}}\left\|y\left(a_{k}\right)\right\|_{H^{-1}(\Omega)^{n}} .
$$

Copyright $@$ ㅇ by SIAM. Unauthorized reproduction of this article is prohibited. 
Using now the estimate of $C_{T}^{\Omega_{1}}$ with respect to $T$ (assumption (1.8)), this leads to

$$
\|v\|_{L^{2}\left(a_{k}, a_{k}+T_{k} ; L^{2}(\partial \Omega)^{m}\right)} \leq c e^{c\left(\frac{1}{T_{k}}+\sqrt{\lambda_{2^{k}}}\right)}\left\|y\left(a_{k}\right)\right\|_{H^{-1}(\Omega)^{n}} .
$$

On the other hand, Weyl's asymptotic formula states that

$$
\sqrt{\lambda_{2^{k}}^{\Omega_{2}}} \underset{+\infty}{\sim} C\left(2^{k}\right)^{\frac{1}{N_{2}}}
$$

and (by the choice of $\rho$ )

$$
\frac{1}{T_{k}}=\frac{1}{M} 2^{k \rho} \leq C 2^{\frac{k}{N_{2}}}
$$

so that

$$
\|v\|_{L^{2}\left(a_{k}, a_{k}+T_{k} ; L^{2}(\partial \Omega)^{m}\right)} \leq C e^{C 2^{\frac{k}{N_{2}}}}\left\|y\left(a_{k}\right)\right\|_{H^{-1}(\Omega)^{n}} .
$$

Combined with (2.4) this yields

$$
\begin{aligned}
\left\|y\left(a_{k}+T_{k}\right)\right\|_{H^{-1}(\Omega)^{n}} & \leq C\left(1+e^{C 2^{\frac{k}{N_{2}}}}\right)\left\|y\left(a_{k}\right)\right\|_{H^{-1}(\Omega)^{n}} \\
& \leq C e^{C 2^{\frac{k}{N_{2}}}}\left\|y\left(a_{k}\right)\right\|_{H^{-1}(\Omega)^{n}} .
\end{aligned}
$$

Step 2: Estimate on the interval $\left[a_{k}+T_{k}, a_{k+1}\right]$. Since $\Pi_{E_{2^{k}}{ }^{-1}} y\left(a_{k}+T_{k}\right)=0$, the dissipation (Proposition 2.4) gives

$$
\left\|y\left(a_{k+1}\right)\right\|_{H^{-1}(\Omega)^{n}} \leq C e^{-\lambda_{2^{k}+1}^{\Omega_{2}} T_{k}}\left\|y\left(a_{k}+T_{k}\right)\right\|_{H^{-1}(\Omega)^{n}} .
$$

Step 3: Final estimate. From (2.7) and (2.6) we deduce

$$
\left\|y\left(a_{k+1}\right)\right\|_{H^{-1}(\Omega)^{n}} \leq C e^{-\lambda_{2^{k}+1}^{\Omega_{2}} T_{k}+C 2^{\frac{k}{N_{2}}}}\left\|y\left(a_{k}\right)\right\|_{H^{-1}(\Omega)^{n}} .
$$

By induction we obtain

$$
\left.\left\|y\left(a_{k+1}\right)\right\|_{H^{-1}(\Omega)^{n}} \leq C e^{\sum_{p=0}^{k}\left(-\lambda_{2^{p}+1}^{\Omega_{2}} T_{p}+C 2^{\frac{p}{N_{2}}}\right.}\right)\left\|y_{0}\right\|_{H^{-1}(\Omega)^{n}} .
$$

Since

$$
\lambda_{2^{p}+1}^{\Omega_{2}} T_{p} \underset{+\infty}{\sim} C\left(2^{p}+1\right)^{\frac{2}{N_{2}}} 2^{-p \rho} \geq C^{\prime}\left(2^{p}\right)^{\frac{2}{N_{2}}-\rho},
$$

we obtain

$$
\left.\left\|y\left(a_{k+1}\right)\right\|_{H^{-1}(\Omega)^{n}} \leq C e^{\sum_{p=0}^{k}\left(-C^{\prime}\left(2^{p}\right)^{\frac{2}{N_{2}}-\rho}+C\left(2^{p}\right)^{\frac{1}{N_{2}}}\right.}\right)\left\|y_{0}\right\|_{H^{-1}(\Omega)^{n}} .
$$

Since $\rho<\frac{1}{N_{2}}$, there exists a $p_{0} \geq 1$ such that

$$
-C^{\prime}\left(2^{p}\right)^{\frac{2}{N_{2}}-\rho}+C\left(2^{p}\right)^{\frac{1}{N_{2}}} \leq-C^{\prime \prime}\left(2^{p}\right)^{\frac{2}{N_{2}}-\rho} \quad \forall p \geq p_{0} .
$$

It follows that, for $k \geq p_{0}$, we have

$$
\sum_{p=0}^{k}\left(-C^{\prime}\left(2^{p}\right)^{\frac{2}{N_{2}}-\rho}+C\left(2^{p}\right)^{\frac{1}{N_{2}}}\right) \leq C^{\prime \prime \prime}-C^{\prime \prime} \sum_{p=p_{0}}^{k}\left(2^{p}\right)^{\frac{2}{N_{2}}-\rho} \leq C^{\prime \prime \prime}-C^{\prime \prime}\left(2^{k}\right)^{\frac{2}{N_{2}}-\rho} .
$$

So that, finally,

$$
\left\|y\left(a_{k+1}\right)\right\|_{H^{-1}(\Omega)^{n}} \leq C e^{-C\left(2^{k}\right)^{\frac{2}{N_{2}}-\rho}}\left\|y_{0}\right\|_{H^{-1}(\Omega)^{n}} .
$$

Copyright (c) by SIAM. Unauthorized reproduction of this article is prohibited. 
Step 4: The function $v$ is a control. Estimates (2.5) and (2.9) show that the function $v$ is in $L^{2}\left(0, T ; L^{2}(\partial \Omega)\right)$ :

$$
\begin{aligned}
\|v\|_{L^{2}\left(0, T: L^{2}(\partial \Omega)^{m}\right)}^{2} & =\sum_{k=0}^{+\infty}\|v\|_{L^{2}\left(a_{k}, a_{k}+T_{k}: L^{2}(\partial \Omega)^{m}\right)}^{2} \\
& \leq C \underbrace{\left(\sum_{k=0}^{+\infty} e^{C 2^{\frac{k}{N_{2}}}-C^{\prime}\left(2^{k}\right)^{\frac{2}{N_{2}}-\rho}}\right)}_{<+\infty \text { by }(2.8)}\left\|y_{0}\right\|_{H^{-1}(\Omega)^{n} .}^{2}
\end{aligned}
$$

Moreover, estimate (2.9) also shows that the function $v$ is indeed a control:

$$
\left\|y\left(a_{k+1}\right)\right\|_{H^{-1}(\Omega)^{n}} \underset{k \rightarrow+\infty}{\longrightarrow} 0=\|y(T)\|_{H^{-1}(\Omega)^{n}} .
$$

3. Cost of the one-dimensional boundary null control. We prove here Theorem 1.4 assuming Theorem 1.5 is proved (see the next section). All along this part we shall use the notation of [AKBGBdT11a].

3.1. Arrangement and properties of the eigenvalues. Let us first recall that the Dirichlet eigenvalues of the Laplacian $-\partial_{x x}^{2}$ on $(0, \pi)$ (with domain $H^{2}(0, \pi) \cap$ $\left.H_{0}^{1}(0, \pi)\right)$ are $\lambda_{k}=k^{2}, k \geq 1$.

We denote by $\left\{\mu_{l}\right\}_{l \in \llbracket 1, p \rrbracket} \subset \mathbb{C}$ the set of distinct eigenvalues of $A^{*}$. For $l \in \llbracket 1, p \rrbracket$, we denote the dimension of the eigenspace of $A^{*}$ associated with $\mu_{l}$ by $n_{l}$ and the size of its Jordan chains by $\tau_{l, j}, j \in \llbracket 1, n_{l} \rrbracket$. In [AKBGBdT11a, Case 2, p. 583], it is shown that we can always assume that $\tau_{l, j}=\tau_{l}$ is independent of $j$. Finally, we set $\widehat{n}=\max _{l \in \llbracket 1, p \rrbracket} n_{l}$.

We assume that the set $\left\{\mu_{l}\right\}_{l \in \llbracket 1, p \rrbracket}$ is arranged in the following (nonunique) way,

$$
\forall l \in \llbracket 1, p-1 \rrbracket \quad\left\{\begin{array}{l}
\Re\left(\mu_{l}\right) \geq \Re\left(\mu_{l+1}\right), \\
\left|\mu_{l}\right| \leq\left|\mu_{l+1}\right| \text { if } \Re\left(\mu_{l}\right)=\Re\left(\mu_{l+1}\right) .
\end{array}\right.
$$

We should point out that in [AKBGBdT11a, p. 562], it is assumed that $\left\{\mu_{l}\right\}_{l \in \llbracket 1, p \rrbracket}$ is ordered in such a way that $\widehat{n}=n_{1}$. Actually, this is only used for convenience and the same reasoning holds if we take $\widehat{n}$ instead of $n_{1}$.

Let us now recall that the eigenvalues of the operator $\partial_{x x}^{2}+A^{*}$ (with domain $\left.H^{2}(0, \pi)^{n} \cap H_{0}^{1}(0, \pi)^{n}\right)$ are given by $-\lambda_{k}+\mu_{i}, k \geq 1$ and $i \in \llbracket 1, p \rrbracket$. Moreover, there exists $k_{0} \geq 1$ such that

$$
-\lambda_{k}+\mu_{i} \neq-\lambda_{l}+\mu_{j}
$$

for every $k \geq k_{0}, l \geq 1, l \neq k$, and $i, j \in \llbracket 1, p \rrbracket$ with $i \neq j$ (see [AKBGBdT11a, Proposition 3.2]).

From (3.1), we see that there exists $k_{1} \geq 1$ large enough so that

$$
2 \lambda_{k_{1}}\left(\Re\left(\mu_{l}\right)-\Re\left(\mu_{l+1}\right)\right)+\left|\mu_{l+1}\right|^{2}-\left|\mu_{l}\right|^{2} \geq 0
$$

for every $l \in \llbracket 1, p-1 \rrbracket$. Therefore, we deduce that

$$
\left|\lambda_{k}-\mu_{l}\right| \leq\left|\lambda_{k}-\mu_{l+1}\right|
$$

for every $k \geq k_{1}$ and $l \in \llbracket 1, p-1 \rrbracket$. 
Finally, let $k_{2} \geq 1$ be large enough so that

$$
1+\left|\lambda_{k}-\mu_{i}\right| \leq\left|\lambda_{k+1}-\mu_{j}\right|
$$

for every $k \geq k_{2}$ and $i, j \in \llbracket 1, p \rrbracket$ with $i \neq j$, which is always possible since $\lambda_{k}=k^{2}$.

We set

$$
K_{0}=\max \left\{k_{0}, k_{1}, k_{2}\right\} .
$$

With this $K_{0}$ we associate $\widetilde{p} \geq 1$, the number of distinct eigenvalues of the matrix $A_{K_{0}}^{*}$ defined in (1.6). Let $\left\{\gamma_{\ell}\right\}_{\ell \in \llbracket 1, \widetilde{p} \rrbracket} \subset\left\{-\lambda_{k}+\mu_{l}\right\}_{k \in \llbracket 1, K_{0} \rrbracket, l \in \llbracket 1, p \rrbracket}$ be the set of distinct eigenvalues of $A_{K_{0}}^{*}$ arranged in such a way that $\left|\gamma_{\ell}\right| \leq\left|\gamma_{\ell+1}\right|$ for every $\ell \in \llbracket 1, \widetilde{p}-1 \rrbracket$.

For $\ell \in \llbracket 1, \widetilde{p} \rrbracket$, the dimension of the eigenspace of $A_{K_{0}}^{*}$ associated with $\gamma_{\ell}$ is denoted by $N_{\ell}$, and the size of its Jordan chains by $\widetilde{\tau}_{\ell, j}, j \in \llbracket 1, N_{\ell} \rrbracket$. Since we assumed that $\tau_{l, j}=\tau_{l}$ it follows that $\widetilde{\tau}_{\ell, j}=\widetilde{\tau}_{\ell}$ is also independent of $j$. Finally, we set $\widehat{N}=\max _{\ell \in \llbracket 1, \widetilde{p} \rrbracket} N_{\ell}$.

We choose to arrange the eigenvalues $\left\{\Lambda_{k}\right\}_{k \geq 1} \subset \mathbb{C}$ of the operator $-\left(\Delta+A^{*}\right)$ as follows:

$$
\left\{\begin{array}{l}
\Lambda_{\ell}=-\gamma_{\ell} \quad \text { for } \ell \in \llbracket 1, \widetilde{p} \rrbracket, \\
\Lambda_{\widetilde{p}+i}=\lambda_{K_{0}+j}-\mu_{l} \quad \text { with } \quad j=\left\lfloor\frac{i-1}{p}\right\rfloor+1 \text { and } l=i-\left\lfloor\frac{i-1}{p}\right\rfloor p \quad \text { for } i \geq 1 .
\end{array}\right.
$$

Observe that the sequence $\left\{\Lambda_{k}\right\}_{k \geq 1}$ satisfies the assumptions 1-5 of Theorem 1.5:

- 1 follows from (3.2);

- 2 holds because the matrix $A$ is stable (see Remark 1);

- 3 is clear since $\left|\Im\left(\Lambda_{k}\right)\right| \leq \max _{l \in \llbracket 1, p \rrbracket}\left|\Im\left(\mu_{l}\right)\right|$ and $\Re\left(\Lambda_{k}\right) \geq \lambda_{1}-\max _{l \in \llbracket 1, p \rrbracket} \Re\left(\mu_{l}\right)$ (which is positive since $A^{*}$ is stable);

- 4 is a consequence of (3.3) and (3.4);

- finally, let us show that 5 holds for $q$ large enough. Let $k=\widetilde{p}+i_{k}$ and $n=\widetilde{p}+i_{n}$ (the case $k \leq \widetilde{p}$ or $n \leq \widetilde{p}$ is simpler). Let $j_{k}, j_{n}$ and $l_{k}, l_{n}$ be such that $\Lambda_{k}=\lambda_{K_{0}+j_{k}}-\mu_{l_{k}}$ and $\Lambda_{n}=\lambda_{K_{0}+j_{n}}-\mu_{l_{n}}$. We have

$$
\begin{aligned}
\left|\Lambda_{n}-\Lambda_{k}\right|^{2}= & \left|\lambda_{K_{0}+j_{k}}-\lambda_{K_{0}+j_{n}}+\mu_{l_{n}}-\mu_{l_{k}}\right|^{2} \\
\geq & || \lambda_{K_{0}+j_{k}}-\lambda_{K_{0}+j_{n}}|-| \mu_{l_{n}}-\mu_{l_{k}}||^{2} \\
\geq & \left|\lambda_{K_{0}+j_{k}}-\lambda_{K_{0}+j_{n}}\right|^{2}-2\left|\lambda_{K_{0}+j_{k}}-\lambda_{K_{0}+j_{n}}\right|\left|\mu_{l_{n}}-\mu_{l_{k}}\right| \\
& +\left|\mu_{l_{n}}-\mu_{l_{k}}\right|^{2} .
\end{aligned}
$$

Let us denote $m=\min _{\substack{\leq l, l^{\prime} \leq p \\ l \neq l^{\prime}}}\left|\mu_{l}-\mu_{l^{\prime}}\right|, \quad M=\max _{\substack{1 \leq l, l^{\prime} \leq p \\ l \neq l^{\prime}}}\left|\mu_{l}-\mu_{l^{\prime}}\right|, d=$ $\left|j_{k}-j_{n}\right|, s=j_{k}+j_{n}$, and $x=d\left(s+2 K_{0}\right)$. Thus,

$$
\left|\Lambda_{n}-\Lambda_{k}\right|^{2} \geq x^{2}-2 M x+m^{2} .
$$

On the other hand, since $\left|i_{k}-i_{n}\right|<p\left(\left|j_{k}-j_{n}\right|+1\right)$ and $i_{k}+i_{n} \leq p\left(j_{k}+j_{n}\right)+2$, we have

$$
\left|k^{2}-n^{2}\right|^{2}=\left|i_{k}-i_{n}\right|^{2}\left(i_{k}+i_{n}+2 \widetilde{p}\right)^{2} \leq p^{2}(d+1)^{2}(s p+2+2 \widetilde{p})^{2} .
$$

By assumption $d, s \rightarrow+\infty$, so that

$$
\left|k^{2}-n^{2}\right|^{2} \leq C d^{2}\left(s+2 K_{0}\right)^{2}=C x^{2} .
$$

Taking, for instance, $\rho=1 / \sqrt{2 C}$ and $x$ large enough we obtain the first property of 5 . The second property is actually satisfied for any $q$. 
The counting function. We recall that the counting function $\mathcal{N}$ associated with the sequence $\left\{\Lambda_{k}\right\}_{k \geq 1}$ is given by

$$
\mathcal{N}(r)=\#\left\{k:\left|\Lambda_{k}\right| \leq r\right\} \quad \forall r>0 .
$$

This function $\mathcal{N}$ is piecewise constant and nondecreasing on the interval $[0,+\infty)$. Thanks to 5 we have $\lim _{k \rightarrow+\infty}\left|\Lambda_{k}\right|=+\infty$, so that $\mathcal{N}(r)<+\infty$ for every $r \in[0,+\infty)$ and $\lim _{r \rightarrow+\infty} \mathcal{N}(r)=+\infty$. Moreover, 4 shows that, for every $r>0$, we have

$$
\mathcal{N}(r)=n \Longleftrightarrow\left(\left|\Lambda_{n}\right| \leq r \text { and }\left|\Lambda_{n+1}\right|>r\right),
$$

so that, in particular, we have

$$
\sqrt{\left|\Lambda_{\mathcal{N}(r)}\right|} \leq \sqrt{r}<\sqrt{\left|\Lambda_{\mathcal{N}(r)+1}\right|}
$$

On the other hand, from the very definition of $\Lambda_{k}$ for $k>\widetilde{p}$, we have

$$
\left(\frac{\mathcal{N}(r)}{p}+\widetilde{K_{0}}\right)^{2}-M \leq\left|\Lambda_{\mathcal{N}(r)}\right| \leq\left(\frac{\mathcal{N}(r)}{p}+\widetilde{\widetilde{K}_{0}}\right)^{2}+M \text { for any } r \text { s.t. } \mathcal{N}(r)>\widetilde{p},
$$

where $M=\max _{l \in \llbracket 1, p \rrbracket}\left|\mu_{l}\right|, \widetilde{K_{0}}=K_{0}-\frac{\widetilde{p}+1}{p}+1$, and $\widetilde{\widetilde{K}_{0}}=\widetilde{K}_{0}+1$. Combining the two previous estimates, it is not difficult to obtain the last assumption 6 of Theorem 1.5.

3.2. The moment problem. In [AKBGBdT11a, Proposition 5.1] it has been proved that, under the assumption (1.5), system (1.9) is null controllable at time $T$ if for every $q \in \llbracket 1, \widehat{N} \rrbracket$ there exists a solution $u_{q} \in L^{2}(0, T)$ to the moments problem (3.6)

$$
\left\{\begin{array}{l}
\int_{0}^{T} \frac{t^{\nu}}{\nu !} e^{\overline{\gamma_{\ell}} t} u_{q}(t) d t=c_{\ell, \nu, q}\left(y_{0} ; T\right) \quad \forall \ell \in \llbracket 1, \widetilde{p} \rrbracket, \forall \nu \in \llbracket 0, \widetilde{\tau}_{\ell}-1 \rrbracket, \\
\int_{0}^{T} \frac{t^{\sigma}}{\sigma !} e^{\left(-\lambda_{k}+\overline{\mu_{l}}\right) t} u_{q}(t) d t=d_{l, \sigma, q}^{k}\left(y_{0} ; T\right) \quad \forall k>K_{0}, \forall l \in \llbracket 1, p \rrbracket, \forall \sigma \in \llbracket 0, \tau_{l}-1 \rrbracket,
\end{array}\right.
$$

where $c_{\ell, \nu, q}$ and $d_{l, \sigma, q}^{k}$ are given in [AKBGBdT11a, Proposition 5.1]. The precise definition of those terms is not really important here, however, we recall that they satisfy the following estimates (see [AKBGBdT11a, equations (49) and (52)]):

$$
\begin{aligned}
\left|c_{\ell, \nu, q}\left(y_{0} ; T\right)\right| & \leq C\left\|e^{A_{K_{0}}^{*} T}\right\|_{\mathcal{M}_{n K_{0}}(\mathbb{C})}\left\|y_{0}\right\|_{H^{-1}(0, \pi)^{n}} \\
& \leq C e^{C T}\left\|y_{0}\right\|_{H^{-1}(0, \pi)^{n}}
\end{aligned}
$$

and

$$
\begin{aligned}
\left|d_{l, \sigma, q}^{k}\left(y_{0} ; T\right)\right| & \leq \frac{C}{k}\left\|e^{\left(-\lambda_{k}+A^{*}\right) T}\right\|_{\mathcal{M}_{n}(\mathbb{C})}\left|\left\langle y_{0}, \phi_{k}\right\rangle_{H^{-1}, H_{0}^{1}(0, \pi)}\right|_{\mathbb{C}^{n}} \\
& \leq C e^{C T} \frac{\sqrt{\lambda_{k}}}{k} e^{-\lambda_{k} T}\left\|y_{0}\right\|_{H^{-1}(0, \pi)^{n}} .
\end{aligned}
$$

The control $v(t)$ is then given as a linear combination of $u_{q}(T-t), q \in \llbracket 1, \widehat{N} \rrbracket$, and as a result satisfies

$$
\|v\|_{L^{2}(0, T)^{m}} \leq C \max _{q \in \llbracket 1, \widehat{N} \rrbracket}\left\|u_{q}\right\|_{L^{2}(0, T)} .
$$

Copyright $@$ by SIAM. Unauthorized reproduction of this article is prohibited. 
Assume for the moment that Theorem 1.5 is proved. Let $T_{0}>0$ be the time given by Theorem 1.5 and set

$$
\eta=\max \left\{\tau_{l}, \widetilde{\tau}_{\ell}, \mid l \in \llbracket 1, p \rrbracket, \ell \in \llbracket 1, \widetilde{p} \rrbracket\right\} .
$$

For $T<T_{0}$ we can then introduce the biorthogonal family $\left\{\varphi_{k, j}\right\}_{k \geq 1, j \in \llbracket 0, \eta-1 \rrbracket} \subset$ $L^{2}(-T / 2, T / 2)$ associated with the sequence $\left\{\Lambda_{k}\right\}_{k \geq 1}$. As we need to work on the interval $(-T / 2, T / 2)$, we perform the change of variable $s=t-\frac{T}{2}$ in (3.6) and obtain

$$
\left\{\begin{aligned}
& \int_{-\frac{T}{2}}^{\frac{T}{2}} \frac{1}{\nu !}\left(s+\frac{T}{2}\right)^{\nu} e^{\overline{\gamma_{\ell}} s} u_{q}\left(s+\frac{T}{2}\right) d s=e^{-\frac{T}{2} \overline{\gamma_{\ell}}} c_{\ell, \nu, q}\left(y_{0} ; T\right) \quad \forall \ell \in \llbracket 1, \widetilde{p} \rrbracket, \forall \nu \in \llbracket 0, \widetilde{\tau}_{\ell}-1 \rrbracket, \\
& \int_{-\frac{T}{2}}^{\frac{T}{2}} \frac{1}{\sigma !}\left(s+\frac{T}{2}\right)^{\sigma} e^{\left(-\lambda_{k}+\overline{\mu_{l}}\right) s} u_{q}\left(s+\frac{T}{2}\right) d s \\
&=e^{-\left(-\lambda_{k}+\overline{\mu_{l}}\right) \frac{T}{2}} d_{l, \sigma, q}^{k}\left(y_{0} ; T\right) \quad\left\{\begin{array}{l}
\forall k>K_{0}, \\
\forall l \in \llbracket 1, p \rrbracket, \\
\forall \sigma \in \llbracket 0, \tau_{l}-1 \rrbracket .
\end{array}\right.
\end{aligned}\right.
$$

Using the binomial formula $\left(s+\frac{T}{2}\right)^{J}=\sum_{j=0}^{J}\left(\begin{array}{c}J \\ j\end{array}\right) s^{J-j}\left(\frac{T}{2}\right)^{j}$ we finally have

$$
\left\{\begin{array}{c}
\sum_{j=0}^{\nu}\left(\begin{array}{l}
\nu \\
j
\end{array}\right)\left(\frac{T}{2}\right)^{j} \int_{-\frac{T}{2}}^{\frac{T}{2}} s^{\nu-j} e^{\overline{\gamma_{\ell}} s} u_{q}\left(s+\frac{T}{2}\right) d s \\
=\widehat{c_{\ell, \nu, q}}\left(y_{0} ; T\right) \quad \forall \ell \in \llbracket 1, \widetilde{p} \rrbracket, \forall \nu \in \llbracket 0, \widetilde{\tau}_{\ell}-1 \rrbracket, \\
\sum_{j=0}^{\sigma}\left(\begin{array}{l}
\sigma \\
j
\end{array}\right)\left(\frac{T}{2}\right)^{j} \int_{-\frac{T}{2}}^{\frac{T}{2}} s^{\sigma-j} e^{\left(-\lambda_{k}+\overline{\mu_{l}}\right) s} u_{q}\left(s+\frac{T}{2}\right) d s \\
=\widehat{d_{l, \sigma, q}^{k}}\left(y_{0} ; T\right) \quad\left\{\begin{array}{l}
\forall k>K_{0}, \\
\forall l \in \llbracket 1, p \rrbracket, \\
\forall \sigma \in \llbracket 0, \tau_{l}-1 \rrbracket
\end{array}\right.
\end{array}\right.
$$

with

$$
\widehat{c_{\ell, \nu, q}}\left(y_{0} ; T\right)=\nu ! e^{-\frac{T}{2} \overline{\gamma_{\ell}}} c_{\ell, \nu, q}\left(y_{0} ; T\right), \quad \widehat{d_{l, \sigma, q}^{k}}\left(y_{0} ; T\right)=\sigma ! e^{-\left(-\lambda_{k}+\overline{\mu_{l}}\right) \frac{T}{2}} d_{l, \sigma, q}^{k}\left(y_{0} ; T\right) .
$$

For $T<T_{0}$, a solution to the moments problem (3.6) is then given for every $t \in(0, T)$ by (note that $-\lambda_{k}+\mu_{l}=-\Lambda_{\widetilde{p}+\left(k-K_{0}-1\right) p+l}$ for $\left.k>K_{0}\right)$

$$
\begin{aligned}
u_{q}(t)=\sum_{\ell=1}^{\widetilde{p}} \sum_{\nu=0}^{\widetilde{\tau}_{\ell}-1} \widehat{\widehat{c_{\ell, \nu, q}}}\left(y_{0} ; T\right) \varphi_{\ell, \nu}\left(t-\frac{T}{2}\right) \\
+\sum_{k>K_{0}} \sum_{l=1}^{p} \sum_{\sigma=0}^{\tau_{l}-1} \widehat{\widehat{d_{l, \sigma, q}^{k}}}\left(y_{0} ; T\right) \varphi_{\widetilde{p}+\left(k-K_{0}-1\right) p+l, \sigma}\left(t-\frac{T}{2}\right),
\end{aligned}
$$

provided that $u_{q}$ lies in $L^{2}(0, T)$ (see below), and where $\widehat{\widehat{c_{\ell, \nu, q}}}$ and $\widehat{\widehat{d_{l, \sigma, q}^{k}}}$ solve the 
triangular systems

$$
P(T)\left(\begin{array}{c}
\widehat{\widehat{c_{\ell, 0, q}}} \\
\vdots \\
\widehat{\widehat{c_{\ell, \tau_{\ell}-1}, q}}
\end{array}\right)=\left(\begin{array}{c}
\widehat{c_{\ell, 0, q}} \\
\vdots \\
\widehat{c_{\ell, \tilde{\tau}_{\ell}-1, q}}
\end{array}\right), \quad Q(T)\left(\begin{array}{c}
\widehat{\widehat{d_{l, 0, q}^{k}}} \\
\vdots \\
\widehat{\widehat{d_{l, \tau_{l}-1, q}^{k}}}
\end{array}\right)=\left(\begin{array}{c}
\widehat{d_{l, 0, q}^{k}} \\
\vdots \\
\widehat{d_{l, \tau_{l}-1, q}^{k}}
\end{array}\right),
$$

where the coefficients of $P(T)$ and $Q(T)$ are, respectively, given for $i \geq j$ by $p_{i j}(T)=$ $\left(\begin{array}{c}i-1 \\ i-j\end{array}\right)\left(\frac{T}{2}\right)^{i-j}, q_{i j}(T)=\left(\begin{array}{c}i-1 \\ i-j\end{array}\right)\left(\frac{T}{2}\right)^{i-j}$, and $p_{i j}(T)=q_{i j}(T)=0$ otherwise. Observe that

$$
\left\|P(T)^{-1}\right\|_{\mathcal{M}_{\tilde{\tau}_{\ell}-1}(\mathbb{C})} \leq C T^{\widetilde{\tau}_{\ell}-1}, \quad\left\|Q(T)^{-1}\right\|_{\mathcal{M}_{\tau_{l}-1}(\mathbb{C})} \leq C T^{\tau_{l}-1}
$$

From this, the definition (3.10) of $\widehat{c_{\ell, \nu, q}}$ and $\widehat{d_{l, \sigma, q}^{k}}$, and the estimates (3.7) and (3.8) of $c_{\ell, \nu, q}$ and $d_{l, \sigma, q}^{k}$, we obtain

$$
\left|\widehat{\widehat{c_{\ell, \nu, q}}}\left(y_{0} ; T\right)\right| \leq C T^{\widetilde{\tau_{\ell}-1}}\left|e^{-\frac{T}{2} \overline{\gamma_{\ell}}}\right| e^{C T}\left\|y_{0}\right\|_{H^{-1}(0, \pi)^{n}} \leq C e^{C T}\left\|y_{0}\right\|_{H^{-1}(0, \pi)^{n}}
$$

and

$$
\begin{aligned}
\left|\widehat{\widehat{d_{l, \sigma, q}^{k}}}\left(y_{0} ; T\right)\right| & \leq C T^{\tau_{l}-1}\left|e^{-\left(-\lambda_{k}+\overline{\mu_{l}}\right) \frac{T}{2}}\right| \frac{\sqrt{\lambda_{k}}}{k} e^{C T} e^{-\lambda_{k} T}\left\|y_{0}\right\|_{H^{-1}(0, \pi)^{n}} \\
& \leq C e^{C T} \frac{\sqrt{\lambda_{k}}}{k} e^{-\lambda_{k} \frac{T}{2}}\left\|y_{0}\right\|_{H^{-1}(0, \pi)^{n}}
\end{aligned}
$$

It remains to prove that $u_{q} \in L^{2}(0, T)$ and to estimate its norm with respect to $T$ and $y_{0}$. It is actually thanks to the estimate (1.12) that this can be achieved. Indeed, using also (3.11) and (3.12) we have

$$
\begin{aligned}
\left\|u_{q}\right\|_{L^{2}(0, T)} \leq & C e^{C T} \sum_{\ell=1}^{\widetilde{p}} e^{C \sqrt{-\Re\left(\gamma_{\ell}\right)}+\frac{C}{T}}\left\|y_{0}\right\|_{H^{-1}(0, \pi)^{n}} \\
& +C e^{C T} \sum_{k>K_{0}} \frac{\sqrt{\lambda_{k}}}{k} e^{-\lambda_{k} \frac{T}{2}} \sum_{l=1}^{p} e^{C \sqrt{\lambda_{k}-\Re\left(\mu_{l}\right)}+\frac{C}{T}}\left\|y_{0}\right\|_{H^{-1}(0, \pi)^{n}} \\
\leq & C e^{C T+\frac{C}{T}}\left(1+\sum_{k>K_{0}} \frac{\sqrt{\lambda_{k}}}{k} e^{-\lambda_{k} \frac{T}{2}+C \sqrt{\lambda_{k}}}\right)\left\|y_{0}\right\|_{H^{-1}(0, \pi)^{n}} .
\end{aligned}
$$

Let us now estimate the series. Young's inequality gives

$$
C \sqrt{\lambda_{k}} \leq \lambda_{k} \frac{T}{4}+\frac{C^{2}}{T}
$$

for every $k \geq 1$ and $T>0$, so that

$$
-\lambda_{k} \frac{T}{2}+C \sqrt{\lambda_{k}} \leq-\lambda_{k} \frac{T}{4}+\frac{C^{2}}{T} .
$$

Thus, using also that $\lambda_{k}=k^{2}$, we obtain

$$
\sum_{k>K_{0}} \frac{\sqrt{\lambda_{k}}}{k} e^{-\lambda_{k} \frac{T}{2}+C \sqrt{\lambda_{k}}} \leq e^{\frac{C}{T}} \sum_{k \geq 0} e^{-k^{2} \frac{T}{4}} .
$$

Copyright $@$ by SIAM. Unauthorized reproduction of this article is prohibited. 
A comparison with the Gauss integral gives

$$
\sum_{k \geq 0} e^{-k^{2} \frac{T}{4}} \leq 2 \sqrt{\frac{4 \pi}{T}} \leq C e^{\frac{C}{T}} .
$$

Coming back to (3.13) we then have

$$
\left\|u_{q}\right\|_{L^{2}(0, T)} \leq C e^{C T+\frac{C}{T}}\left\|y_{0}\right\|_{H^{-1}(0, \pi)^{n}} .
$$

Finally, (3.9) gives, for every $T<T_{0}$,

$$
\|v\|_{L^{2}(0, T)} \leq C e^{\frac{C}{T}}\left\|y_{0}\right\|_{H^{-1}(0, \pi)^{n}} .
$$

Thus, when $T<T_{0}$ we have obtained a null control to system (1.9) which satisfies the desired estimate. The case $T \geq T_{0}$ is actually reduced to the previous one. Indeed, any continuation by zero of a control on $\left(0, T_{0} / 2\right)$ is a control on $(0, T)$ and the estimate follows from the decrease of the cost with respect to the time.

4. Biorthogonal families to complex matrix exponentials. This section is devoted to the proof of Theorem 1.5.

4.1. Idea of the proof. For $T$ small enough and any $\eta \geq 1$, we have to construct a family $\left\{\varphi_{k, j}\right\}_{k \geq 1, j \in \llbracket 0, \eta-1 \rrbracket}$ in $L^{2}(-T / 2, T / 2)$ such that

$$
\int_{-\frac{T}{2}}^{\frac{T}{2}} \varphi_{k, j}(t) t^{\nu} e^{-\overline{\Lambda_{l}} t} d t=\delta_{k l} \delta_{j \nu}
$$

for every $k, l \geq 1$ and $j, \nu \in \llbracket 0, \eta-1 \rrbracket$, with in addition the following bound,

$$
\left\|\varphi_{k, j}\right\|_{L^{2}\left(-\frac{T}{2}, \frac{T}{2}\right)} \leq C e^{C \sqrt{\Re\left(\Lambda_{k}\right)}+\frac{C}{T}}
$$

for any $k \geq 1$ and $j \in \llbracket 0, \eta-1 \rrbracket$.

The idea is to use the Fourier transform with the help of the Paley-Wiener theorem (see [Rud74, Theorem 19.3]) that we recall here.

THEOREM 4.1. Let $\Phi$ be an entire function of exponential type $T / 2$ (that is ${ }^{3}$ $|\Phi(z)| \leq C e^{\frac{T}{2}|z|}$ for all $\left.z \in \mathbb{C}\right)$ such that

$$
\|\Phi\|_{L^{2}(-\infty,+\infty)}^{2}=\int_{-\infty}^{+\infty}|\Phi(x)|^{2} d x<+\infty .
$$

Then, there exists $\varphi \in L^{2}(-T / 2, T / 2)$ such that

$$
\Phi(z)=\frac{1}{\sqrt{2 \pi}} \int_{-\frac{T}{2}}^{\frac{T}{2}} \varphi(t) e^{i t z} d t \quad \forall z \in \mathbb{C} .
$$

Moreover, the Plancherel theorem gives

$$
\|\varphi\|_{L^{2}\left(-\frac{T}{2}, \frac{T}{2}\right)}=\|\Phi\|_{L^{2}(-\infty,+\infty)} .
$$

\footnotetext{
${ }^{3}$ Here and only here, $C$ may even depend on $T$ without affecting the result.
}

Copyright $\odot$ by SIAM. Unauthorized reproduction of this article is prohibited. 
Observe that the function in (4.1) is infinitely differentiable on $\mathbb{C}$ with, for every $\nu \in \llbracket 0, \eta-1 \rrbracket$,

$$
\Phi^{(\nu)}(z)=\frac{i^{\nu}}{\sqrt{2 \pi}} \int_{-\frac{T}{2}}^{\frac{T}{2}} \varphi(t) t^{\nu} e^{i t z} d t \quad \forall z \in \mathbb{C} .
$$

Thus, Theorem 1.5 will be proved if we manage to build suitable entire functions as stated in the following result.

THEOREM 4.2. Assume that the sequence $\left\{\Lambda_{k}\right\}_{k \geq 1} \subset \mathbb{C}$ satisfies the assumptions $1-6$.

There exists $T_{0}>0$ such that, for any $\eta \geq 1$ and $0<T<T_{0}$, there exists a family $\left\{\Phi_{k, j}\right\}_{k \geq 1, j \in \llbracket 0, \eta-1 \rrbracket}$ of entire functions of exponential type $T / 2$ satisfying

$$
\Phi_{k, j}^{(\nu)}\left(i \overline{\Lambda_{l}}\right)=\frac{i^{\nu}}{\sqrt{2 \pi}} \delta_{k l} \delta_{j \nu} \quad \forall k, l \geq 1, \quad \forall j, \nu \in \llbracket 0, \eta-1 \rrbracket,
$$

and

$$
\left\|\Phi_{k, j}\right\|_{L^{2}(-\infty,+\infty)} \leq C e^{C \sqrt{\Re\left(\Lambda_{k}\right)}+\frac{C}{T}}
$$

for any $k \geq 1$ and $j \in \llbracket 0, \eta-1 \rrbracket$.

Remark 3. A sequence $\left\{\Lambda_{k}\right\}_{k \geq 1} \subset \mathbb{C}$ satisfies the assumptions 1-6 if and only if so does the sequence $\left\{\overline{\Lambda_{k}}\right\}_{k \geq 1}$. For this reason, we will prove Theorem 4.2 for the sequence $\left\{\overline{\Lambda_{k}}\right\}_{k \geq 1}$.

\subsection{Proof of Theorem 4.2.}

Some preliminary remarks. It is interesting to point out some properties of the sequence $\left\{\Lambda_{k}\right\}_{k \geq 1}$ which can be deduced from assumptions 3,4 , and 6 .

1. First, under assumptions 4 and 6 we have that

$$
\sum_{k \geq 1} \frac{1}{\left|\Lambda_{k}\right|}<+\infty
$$

Indeed, using that $\mathcal{N}$ is piecewise constant and nondecreasing on the interval $[0,+\infty)$, we can write

$$
\begin{aligned}
\sum_{k \geq 1} \frac{1}{\left|\Lambda_{k}\right|} & =\int_{\left|\Lambda_{1}\right|^{-}}^{+\infty} \frac{1}{r} d \mathcal{N}(r)=\int_{\left|\Lambda_{1}\right|}^{+\infty} \frac{1}{r^{2}} \mathcal{N}(r) d r \\
& \leq \int_{\left|\Lambda_{1}\right|}^{+\infty} \frac{\alpha+p \sqrt{r}}{r^{2}} d r=\frac{\alpha}{\left|\Lambda_{1}\right|}+\frac{2 p}{\sqrt{\left|\Lambda_{1}\right|}}<+\infty
\end{aligned}
$$

2. Then, from assumption 3 we can also deduce the following behavior of the sequence $\left\{\Lambda_{k}\right\}_{k \geq 1}$ :

$$
\left|\Lambda_{k}\right|-\Re\left(\Lambda_{k}\right) \leq \beta \sqrt{\Re\left(\Lambda_{k}\right)} \quad \text { and } \quad\left|\Lambda_{k}\right| \leq C \Re\left(\Lambda_{k}\right) \quad \forall k \geq 1 .
$$

Indeed, one has

$$
\left|\Lambda_{k}\right|^{2}=\Re\left(\Lambda_{k}\right)^{2}+\Im\left(\Lambda_{k}\right)^{2} \leq \Re\left(\Lambda_{k}\right)^{2}+\beta^{2} \Re\left(\Lambda_{k}\right) \leq\left(\Re\left(\Lambda_{k}\right)+\beta \sqrt{\Re\left(\Lambda_{k}\right)}\right)^{2} .
$$

Copyright (c) by SIAM. Unauthorized reproduction of this article is prohibited. 
Let us now introduce the complex functions given, for every $z \in \mathbb{C}$, by

$$
f(z)=\prod_{k \geq 1}\left(1-\frac{z}{\Lambda_{k}}\right), \quad f_{n}(z)=\prod_{\substack{k \geq 1 \\ k \neq n}}\left(1-\frac{z}{\Lambda_{k}}\right) .
$$

Thanks to (4.4), the previous products are uniformly convergent on compact sets of $\mathbb{C}$ and therefore $f$ and $f_{n}$ are entire functions. Moreover, the zeros of $f$ and $f_{n}$ are exactly $\left\{\Lambda_{k}\right\}_{k \geq 1}$ and $\left\{\Lambda_{k}\right\}_{k \neq n}$ and they are zeros of multiplicity 1 (recall that the $\Lambda_{k}$ are distinct by 1). For a proof of these facts we refer to [Rud74, Theorem 15.4].

On the other hand, let us fix $d=p \pi+2$. For any $\tau>0$ such that $\tau<d^{2} / 2$ we define the real positive sequence $\left\{a_{n}\right\}_{n \geq 0}$ given by

$$
a_{n}=\frac{d^{2}}{\tau^{2}}+\frac{4\left(n^{2}-1\right)}{d^{2}} \quad \forall n \geq 0 .
$$

With this sequence we associate a complex function $M$ defined by

$$
M(z)=\prod_{n \geq 1} \frac{\sin \left(z / a_{n}\right)}{z / a_{n}} \quad \forall z \in \mathbb{C} .
$$

Since

$$
\left|\frac{\sin (z)}{z}\right| \leq e^{|z|} \quad \forall z \in \mathbb{C}
$$

and $a_{n} \underset{+\infty}{\sim} C n^{2}$, the previous product is uniformly convergent on compact sets of $\mathbb{C}$ and $M$ is an entire function of exponential type $\tau_{M}>0$, where

$$
\tau_{M}=\sum_{n \geq 1} \frac{1}{a_{n}}<+\infty
$$

More precisely, $M$ satisfies

$$
|M(z)| \leq e^{\tau_{M}|z|} \quad \forall z \in \mathbb{C} .
$$

Observe that there is no constant in front of the term $e^{\tau_{M}|z|}$. This point will be very important in what follows (see the proof of Proposition 4.3 in Appendix A) to obtain estimates with constants $C$ that do not depend on $\tau$ (which will play the role of $T$; see below). Note also that $M$ has only real zeros since $\left\{a_{n}\right\}_{n \geq 1}$ is a real sequence. Finally, we will often use that $\tau_{M}<\tau$. This fact is proved in Lemma A.2 in Appendix A.

Proof of Theorem 4.2. We follow some techniques developed in [AKBGBdT11a] (see, in particular, Lemma 4.4 in this reference).

Set $T_{0}=d^{2}$ and, for any $0<T<T_{0}$, set $\tau=\frac{T}{2 \eta}$ in such a way that the condition $\tau<d^{2} / 2$ holds. The function $M$ defined above will then correspond to this value of $\tau$.

Let us consider the functions

$$
\left\{\begin{array}{cl}
\Phi_{k}(z)=\frac{1}{\eta !}\left[W_{k}(z)\right]^{\eta}, & W_{k}(z)=\frac{f(-i z)}{-i f^{\prime}\left(\Lambda_{k}\right)} \frac{M\left(z+\Im\left(\Lambda_{k}\right)\right)}{M\left(i \Re\left(\Lambda_{k}\right)\right)} \\
\widetilde{\Phi}_{k}(z)=\frac{1}{\eta !}\left[\widetilde{W}_{k}(z)\right]^{\eta}, & \widetilde{W}_{k}(z)=\frac{f_{k}(-i z)}{-i f^{\prime}\left(\Lambda_{k}\right)} \frac{M\left(z+\Im\left(\Lambda_{k}\right)\right)}{M\left(i \Re\left(\Lambda_{k}\right)\right)},
\end{array}\right.
$$

defined for every $z \in \mathbb{C}$ and $k \geq 1$. 
Let us first give some estimates for the functions $W_{k}, \widetilde{W}_{k}$ (and, as a result, also for $\Phi_{k}$ and $\widetilde{\Phi}_{k}$ ) that will be used later.

Proposition 4.3. Assume that the sequence $\left\{\Lambda_{k}\right\}_{k \geq 1}$ satisfies the assumptions $1-6$, and let $\tau<d^{2} / 2$. Then, for any $k \geq 1$ and $z \in \mathbb{C}$,

$$
\left|W_{k}(z)\right|+\left|\widetilde{W}_{k}(z)\right| \leq e^{C \sqrt{|z|}+\tau_{M}\left(|z|-\Re\left(\Lambda_{k}\right)\right)+C \sqrt{\Re\left(\Lambda_{k}\right)}+\frac{C}{\tau}} .
$$

On the other hand, for any $k \geq 1$ and $x \in \mathbb{R}$,

$$
\left|W_{k}(x)\right|+\left|\widetilde{W}_{k}(x)\right| \leq e^{-\sqrt{|x|}+C \sqrt{\Re\left(\Lambda_{k}\right)}+\frac{C}{\tau}} .
$$

The proof of this rather technical proposition is given in Appendix A. For now, let us continue with the proof of Theorem 4.2.

Since the function $M$ only has real zeros, all the functions introduced in (4.11) are well-defined and they are entire functions. For every $l \geq 1, i \Lambda_{l}$ is a simple zero of the function $W_{k}$ since $\Lambda_{l}$ is a simple zero of $f$ and $i \Lambda_{l}+\Im\left(\Lambda_{k}\right)$ is not a zero of $M\left(\Im\left[i \Lambda_{l}+\Im\left(\Lambda_{k}\right)\right]=\Re\left(\Lambda_{l}\right) \neq 0\right.$ by assumption 2). Thus, we deduce that, for every $l \geq 1, i \Lambda_{l}$ is a zero of $\Phi_{k}$ with exact multiplicity $\eta$, i.e.,

$$
\Phi_{k}^{(\eta)}\left(i \Lambda_{l}\right)=\left[W_{k}^{\prime}\left(i \Lambda_{l}\right)\right]^{\eta} \neq 0 \quad \text { and } \quad \Phi_{k}^{(\nu)}\left(i \Lambda_{l}\right)=0 \quad \forall k, l \geq 1, \quad \forall \nu \in \llbracket 0, \eta-1 \rrbracket .
$$

Observe that, in particular $\Phi_{k}^{(\eta)}\left(i \Lambda_{k}\right)=1$. At this point, the function $\Phi_{k, j}=\Phi_{k}$ then satisfies (4.2) for $l \neq k$.

For any $k \geq 1, j \in \llbracket 0, \eta-1 \rrbracket$, and $z \in \mathbb{C}$, let us now set

$$
f_{k, j}(z)=\frac{\Phi_{k}(z)}{\left(z-i \Lambda_{k}\right)^{\eta-j}}=\left(\frac{-1}{i \Lambda_{k}}\right)^{\eta} \widetilde{\Phi}_{k}(z)\left(z-i \Lambda_{k}\right)^{j} .
$$

Note that, for $x \in \mathbb{R}$, we deduce from (4.13), assumption 4, and (4.5), that

$$
\left|f_{k, j}(x)\right| \leq C e^{-\frac{\eta}{2} \sqrt{|x|}+C \sqrt{\Re\left(\Lambda_{k}\right)}+\frac{C}{\tau}} .
$$

From the properties of the function $\Phi_{k}$, we get

$$
\begin{cases}f_{k, j}^{(\nu)}\left(i \Lambda_{l}\right)=0 & \forall l \geq 1 \text { with } l \neq k, \forall \nu \in \llbracket 0, \eta-1 \rrbracket \\ f_{k, j}^{(\nu)}\left(i \Lambda_{k}\right)=0 & \forall \nu \in \llbracket 0, j-1 \rrbracket, \\ f_{k, j}^{(j+r)}\left(i \Lambda_{k}\right)=\frac{(j+r) !}{(\eta+r) !} \Phi_{k}^{(\eta+r)}\left(i \Lambda_{k}\right) & \forall r \geq 0\end{cases}
$$

We look now for $\Phi_{k, j}$ in the following form:

$$
\Phi_{k, j}(z)=p(z) f_{k, j}(z)
$$

with $p$ a polynomial function of degree $\eta-j-1$ which depends on $k, j$ (for simplicity, this dependence is omitted in the notation).

As a consequence of inequality (4.12) and the fact that $\tau_{M}<\tau$, the function $\Phi_{k, j}$ is an entire function of exponential type $\eta \tau=T / 2{ }^{4}$

\footnotetext{
${ }^{4}$ The constant $C$ such that $\left|\Phi_{k, j}(z)\right| \leq C e^{\eta \tau|z|}$ for every $z \in \mathbb{C}$ depends on $k, j, \tau$, etc., but this is not important as mentioned earlier.
}

Copyright (C) by SIAM. Unauthorized reproduction of this article is prohibited. 
In view of (4.15), if we simply take $p=1$, then the relations (4.2) are satisfied for $l \neq k$ and $l=k$ if $\nu<j$. Thus, in order to get (4.2), we have to choose $p$ such that $\Phi_{k, j}^{(j)}\left(i \Lambda_{k}\right)=\frac{i^{j}}{\sqrt{2 \pi}}$ and $\Phi_{k, j}^{(j+r)}\left(i \Lambda_{k}\right)=0$ for $r \in \llbracket 1, \eta-j-1 \rrbracket$, that is,

$$
\left\{\begin{array}{l}
p\left(i \Lambda_{k}\right)=\frac{i^{j}}{\sqrt{2 \pi}} \frac{1}{f_{k, j}^{(j)}\left(i \Lambda_{k}\right)}=\frac{i^{j}}{\sqrt{2 \pi}} \frac{\eta !}{j !}, \\
\sum_{\ell=0}^{r-1} a_{r \ell} p^{(\ell)}\left(i \Lambda_{k}\right)+p^{(r)}\left(i \Lambda_{k}\right)=0 \quad \forall r \in \llbracket 1, \eta-j-1 \rrbracket
\end{array}\right.
$$

where

$$
a_{r \ell}=\frac{\left(\begin{array}{c}
j+r \\
l
\end{array}\right)}{\left(\begin{array}{c}
j+r \\
r
\end{array}\right)} \frac{f_{k, j}^{(j+r-\ell)}\left(i \Lambda_{k}\right)}{f_{k, j}^{(j)}\left(i \Lambda_{k}\right)}=\frac{r ! \eta !}{\ell !(\eta+r-\ell) !} \Phi_{k}^{(\eta+r-\ell)}\left(i \Lambda_{k}\right)
$$

for every $r \in \llbracket 1, \eta-j-1 \rrbracket$ and $\ell \in \llbracket 0, r-1 \rrbracket$ (they are well-defined since $f_{k, j}^{(j)}\left(i \Lambda_{k}\right) \neq 0$ ).

These relations allow us to compute $p^{(r)}\left(i \Lambda_{k}\right)$ for every $r \in \llbracket 0, \eta-j-1 \rrbracket$ and thus completely determine $p$ which is then given by

$$
p(z)=\sum_{r=0}^{\eta-j-1} \frac{p^{(r)}\left(i \Lambda_{k}\right)}{r !}\left(z-i \Lambda_{k}\right)^{r} .
$$

In order to get the bound (4.3) for $\Phi_{k, j}$, let us prove some estimates of the polynomial $p$ previously constructed. If we set $P=\left(p^{(r)}\left(i \Lambda_{k}\right)\right)_{r \in \llbracket 0, \eta-j-1 \rrbracket} \in \mathbb{C}^{\eta-j}$, then we can rewrite the identities in (4.16) as a linear system of the form $\mathbf{A} P=\mathbf{B}$ with

$$
\begin{aligned}
\mathbf{A} & =\left(\begin{array}{ccccc}
1 & 0 & \cdots & \cdots & 0 \\
a_{10} & 1 & \ddots & & \vdots \\
a_{20} & a_{21} & \ddots & \ddots & \vdots \\
\vdots & \vdots & \ddots & \ddots & 0 \\
a_{\eta-j-1,0} & a_{\eta-j-1,1} & \cdots & a_{\eta-j-1, \eta-j-2} & 1
\end{array}\right) \in \mathcal{M}_{\eta-j}(\mathbb{C}) \\
\mathbf{B} & =\left(\begin{array}{c}
\frac{i^{j}}{\sqrt{2 \pi}} \frac{\eta !}{j !} \\
0 \\
\vdots \\
\vdots \\
0
\end{array}\right) \in \mathbb{C}^{\eta-j},
\end{aligned}
$$

and $a_{r \ell}$ given in (4.17). Again, following [AKBGBdT11a, eq. (31), p. 570], it is possible to show

$$
|P|_{\mathbb{C}^{\eta-j}} \leq C\left(\sum_{\substack{r \in \llbracket 1, \eta-j-1 \rrbracket \\ \ell \in \llbracket 0, r \rrbracket}}\left|\Phi_{k}^{(\eta+r-\ell)}\left(i \Lambda_{k}\right)\right|^{2}\right)^{\frac{\eta-j-1}{2}} .
$$

Copyright (c) by SIAM. Unauthorized reproduction of this article is prohibited. 
Finally, let us estimate $\left|\Phi_{k}^{(\eta+r-\ell)}\left(i \Lambda_{k}\right)\right|$, for $r \in \llbracket 1, \eta-j-1 \rrbracket$ and $\ell \in \llbracket 0, r \rrbracket$. Since $\Phi_{k}$ is an entire function, we can write

$$
\Phi_{k}^{(m)}\left(i \Lambda_{k}\right)=\frac{m !}{2 i \pi} \int_{\left|z-i \Lambda_{k}\right|=1} \frac{\Phi_{k}(z)}{\left(z-i \Lambda_{k}\right)^{m+1}} d z \quad \forall m \geq 0,
$$

so that

$$
\left|\Phi_{k}^{(m)}\left(i \Lambda_{k}\right)\right| \leq C \sup _{z:\left|z-i \Lambda_{k}\right|=1}\left|\Phi_{k}(z)\right| .
$$

Using inequality (4.12), the fact that $|z| \leq 1+\left|\Lambda_{k}\right|$ for $z$ such that $\left|z-i \Lambda_{k}\right|=1$, inequalities (4.5), and the fact that $\tau_{M}<d^{2} / 2$, we obtain

$$
\left|\Phi_{k}^{(m)}\left(i \Lambda_{k}\right)\right| \leq C e^{C \sqrt{\Re\left(\Lambda_{k}\right)}+\frac{C}{\tau}} \quad \forall k \geq 1, \forall m \geq 0 .
$$

Going back to (4.18), we get

$$
|P|_{\mathbb{C}^{\eta-j}} \leq C e^{C \sqrt{\Re\left(\Lambda_{k}\right)}+\frac{C}{\tau}} .
$$

Recall that the vector $P$ contains the coefficients $p^{(r)}\left(i \Lambda_{k}\right)$ of the polynomial $p$. Thus, using that $|z|^{r} / r ! \leq C e^{\frac{\eta}{4} \sqrt{|z|}}$ for any $r \in \llbracket 0, \eta \rrbracket$, and using (4.5), we obtain

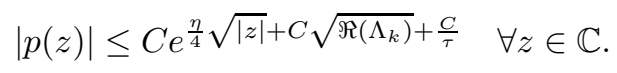

Combining the previous estimate, written for $x \in \mathbb{R}$, and (4.14) we deduce the expected bound (4.3) for $\Phi_{k, j}=p f_{k, j}$.

Appendix A. Proof of Proposition 4.3. We start with another property satisfied by the sequence $\left\{\Lambda_{k}\right\}_{k \geq 1}$, namely, that it behaves as $k^{2}$.

Lemma A.1. Under assumptions 4, 5, and 6, we have

$$
C k \leq \sqrt{\left|\Lambda_{k}\right|} \leq C^{\prime} k \quad \forall k \geq 1 .
$$

The second lemma was often used.

Lemma A.2. Let $\tau<d^{2} / 2$. For the function $M$ given by (4.8) we have $\tau_{M}<\tau$ (where $\tau_{M}$ is given in (4.9)).

The next lemmas are devoted to giving bounds of every term involved in the definitions (4.11) of $W_{k}$ and $\widetilde{W}_{k}$.

Lemma A.3. Under assumption 6 we have, for every $z \in \mathbb{C}$ and $n \geq 1$,

$$
\log |f(z)| \leq(d-1) \sqrt{|z|}+C, \quad \log \left|f_{n}(z)\right| \leq(d-1) \sqrt{|z|}+C,
$$

where $f$ and $f_{n}$ are defined in (4.6).

LemmA A.4. Under assumptions 4, 5, and 6 we have, for every $n \geq 1$,

$$
\log \left|f^{\prime}\left(\Lambda_{n}\right)\right| \geq-C \sqrt{\left|\Lambda_{n}\right|}
$$

where $f$ is defined in (4.6).

Lemma A.5. Let $\tau<d^{2} / 2$. The function $M$ given by (4.8) satisfies

$$
M(0)=1, \quad \log |M(x)| \leq-d \sqrt{|x|}+\frac{C}{\tau} \quad \forall x \in \mathbb{R} .
$$

Copyright $@$ by SIAM. Unauthorized reproduction of this article is prohibited. 
Lemma A.6. Let $\tau<d^{2} / 2$. The function $M$ given by (4.8) satisfies

$$
\log |M(i y)| \geq 0 \quad \forall y \in \mathbb{R}
$$

and also

$$
\log |M(i y)| \geq \tau_{M}|y|-C \sqrt{|y|}-\frac{C}{\tau} \quad \forall y \in \mathbb{R} .
$$

Proof of Proposition 4.3. Let us recall the definition of $W_{k}$ :

$$
W_{k}(z)=\frac{f(-i z)}{-i f^{\prime}\left(\Lambda_{k}\right)} \frac{M\left(z+\Im\left(\Lambda_{k}\right)\right)}{M\left(i \Re\left(\Lambda_{k}\right)\right)} .
$$

From Lemmas A.3 and A.4 and $\left|\Lambda_{k}\right| \leq C \Re\left(\Lambda_{k}\right)$ (see (4.5)) we deduce that

$$
\left|\frac{f(-i z)}{-i f^{\prime}\left(\Lambda_{k}\right)}\right| \leq e^{(d-1) \sqrt{|z|}+C \sqrt{\Re\left(\Lambda_{k}\right)}} .
$$

On the other hand, from inequality (A.4) of Lemma A.6 and using (4.10) we can also infer

$$
\left|\frac{M\left(z+\Im\left(\Lambda_{k}\right)\right)}{M\left(i \Re\left(\Lambda_{k}\right)\right)}\right| \leq e^{\tau_{M}|z|+\tau_{M}\left(\left|\Im\left(\Lambda_{k}\right)\right|-\Re\left(\Lambda_{k}\right)\right)+C \sqrt{\Re\left(\Lambda_{k}\right)}+\frac{C}{\tau}} .
$$

Note that $\tau_{M}\left|\Im\left(\Lambda_{k}\right)\right| \leq C \sqrt{\Re\left(\Lambda_{k}\right)}$ thanks to assumption 3 and $\tau_{M}<d^{2} / 2$. Thus, putting both inequalities together we deduce estimate (4.12) for the function $W_{k}$.

Let us now take $x \in \mathbb{R}$. Applying inequality (A.2) of Lemma A.5 and, this time, inequality (A.3) of Lemma A.6, we arrive at

$$
\left|\frac{M\left(x+\Im\left(\Lambda_{k}\right)\right)}{M\left(i \Re\left(\Lambda_{k}\right)\right)}\right| \leq e^{-d \sqrt{|x|}+d \sqrt{\left|\Im\left(\Lambda_{k}\right)\right|}+\frac{C}{\tau}}
$$

Note that $\sqrt{\left|\Im\left(\Lambda_{k}\right)\right|} \leq C \sqrt{\Re\left(\Lambda_{k}\right)}$ by (4.5). Thus, the previous inequality together with (A.5) (written for $x \in \mathbb{R}$ ) provide the estimate (4.13) for $W_{k}(x)$, with $x$ real.

The same reasoning provides the estimate for $\widetilde{W}_{k}$.

Proof of Lemma A.1. The lower bound easily follows from assumption 5 by taking $n=1$.

To prove the upper bound, let us first observe that, for any $k$ and $n$ such that $\left|\Lambda_{k}\right|=\left|\Lambda_{n}\right|$, we have, using assumption 4 ,

$$
\left|\Re\left(\Lambda_{k}\right)^{2}-\Re\left(\Lambda_{n}\right)^{2}\right|=\left|\Im\left(\Lambda_{k}\right)^{2}-\Im\left(\Lambda_{n}\right)^{2}\right| \leq \beta^{2}\left(\Re\left(\Lambda_{k}\right)+\Re\left(\Lambda_{n}\right)\right),
$$

so that

$$
\left|\Re\left(\Lambda_{k}\right)-\Re\left(\Lambda_{n}\right)\right| \leq \beta^{2} .
$$

It follows that (using assumption 4 again)

$$
\left|\Lambda_{k}-\Lambda_{n}\right| \leq\left|\Re\left(\Lambda_{k}\right)-\Re\left(\Lambda_{n}\right)\right|+\left|\Im\left(\Lambda_{k}\right)-\Im\left(\Lambda_{n}\right)\right| \leq \beta^{2}+2 \beta \sqrt{\left|\Lambda_{k}\right|} .
$$

By using assumption 5 , and the fact that $k+n \geq k$, we obtain

$$
|k-n| \leq \max \left\{q, \frac{\beta^{2}+2 \beta \sqrt{\left|\Lambda_{k}\right|}}{\rho k}\right\} .
$$

Copyright $@$ by SIAM. Unauthorized reproduction of this article is prohibited. 
Note that if $k$ is such that $\frac{\beta^{2}+2 \beta \sqrt{\left|\Lambda_{k}\right|}}{\rho k} \leq q$ then $\sqrt{\left|\Lambda_{k}\right|} \leq\left(\frac{q \rho}{2 \beta}\right) k$ and we are done. Let us then deal with the $k$ such that $\frac{\beta^{2}+2 \beta \sqrt{\left|\Lambda_{k}\right|}}{\rho k}>q$.

Applying the previous estimate with $n=\mathcal{N}\left(\left|\Lambda_{k}\right|\right)$ (which indeed satisfies $\left|\Lambda_{n}\right|=$ $\left|\Lambda_{k}\right|$ by (3.5) and assumption 4$)$, we deduce that

$$
\mathcal{N}\left(\left|\Lambda_{k}\right|\right) \leq k+\left|\mathcal{N}\left(\left|\Lambda_{k}\right|\right)-k\right| \leq k+\frac{\beta^{2}+2 \beta \sqrt{\left|\Lambda_{k}\right|}}{\rho k},
$$

and by assumption 6 we finally obtain

$$
p \sqrt{\left|\Lambda_{k}\right|} \leq \alpha+\mathcal{N}\left(\left|\Lambda_{k}\right|\right) \leq k+\frac{\beta^{2}+2 \beta \sqrt{\left|\Lambda_{k}\right|}}{\rho k} .
$$

For $k$ large enough, we obtain

$$
\frac{p}{2} \sqrt{\left|\Lambda_{k}\right|} \leq k+\frac{\beta^{2}}{\rho k} \leq\left(1+\frac{\beta^{2}}{\rho}\right) k,
$$

and the lemma is proved.

Proof of Lemma A.2. For the proof we will follow some ideas from [FR71] and [Mil04] (see also [Red77]). Let us consider the counting function $N$ associated with the sequence $\left\{a_{n}\right\}_{n \geq 1}$ given by (4.7):

$$
N(r)=\#\left\{n \geq 1: a_{n} \leq r\right\}
$$

Observe that the sequence $\left\{a_{n}\right\}_{n \geq 0}$ can be written as

$$
a_{n}=a_{0}+\frac{n^{2}}{A^{2}} \quad \forall n \geq 1 \quad \text { with } \quad A=\frac{d}{2} \quad \text { and } \quad a_{0}=\frac{d^{2}}{\tau^{2}}-\frac{4}{d^{2}}
$$

and that $a_{0}>0$ since we assumed that $\tau<d^{2} / 2$. Thus, $N(r)=0$ for $r<a_{1}$ and

$$
N(r)=\left\lfloor A \sqrt{r-a_{0}}\right\rfloor \quad \forall r \geq a_{1},
$$

where we recall that $L \cdot\rfloor$ is the floor function. Note that

$$
A \sqrt{r}-A \sqrt{a_{0}} \leq N(r) \leq A \sqrt{r} \quad \forall r \geq 0 .
$$

These remarks in mind, we have

$$
\begin{aligned}
\tau_{M} & =\sum_{n \geq 1} \frac{1}{a_{n}}=\int_{a_{1}^{-}}^{+\infty} \frac{1}{r} d N(r)=\int_{a_{1}^{-}}^{+\infty} \frac{N(r)}{r^{2}} d r \leq \int_{a_{1}}^{+\infty} \frac{A \sqrt{r-a_{0}}}{r^{2}} d r \\
& <A \int_{a_{1}}^{+\infty} \frac{\sqrt{r}}{r^{2}} d r=\frac{2 A}{\sqrt{a_{1}}}=\tau
\end{aligned}
$$

where the last inequality is strict since $a_{0} \neq 0$.

Proof of Lemma A.3. Given $z \in \mathbb{C}$, one has

$$
\log |f(z)| \leq \sum_{k \geq 1} \log \left(1+\frac{|z|}{\left|\Lambda_{k}\right|}\right)=\int_{\left|\Lambda_{1}\right|^{-}}^{+\infty} \log \left(1+\frac{|z|}{t}\right) d \mathcal{N}(t) .
$$

Copyright (C) by SIAM. Unauthorized reproduction of this article is prohibited. 
Taking into account $\lim _{t \rightarrow+\infty} \mathcal{N}(t) / t=0$ (consequence of assumption 6) an integration by parts gives

$$
\int_{\left|\Lambda_{1}\right|^{-}}^{+\infty} \log \left(1+\frac{|z|}{t}\right) d \mathcal{N}(t)=\int_{\left|\Lambda_{1}\right|^{-}}^{+\infty} \frac{|z|}{t(|z|+t)} \mathcal{N}(t) d t
$$

After the change of variable $t=|z| s$, we obtain

$$
\int_{\left|\Lambda_{1}\right|^{-}}^{+\infty} \frac{|z|}{t(|z|+t)} \mathcal{N}(t) d t=\int_{\left|\Lambda_{1}\right|^{-} /|z|}^{+\infty} \frac{\mathcal{N}(|z| s)}{s(s+1)} d s
$$

From assumption 6, we conclude that

$$
\begin{aligned}
\int_{\left|\Lambda_{1}\right|^{-} /|z|}^{+\infty} \frac{\mathcal{N}(|z| s)}{s(s+1)} d s & \leq p \sqrt{|z|} \int_{\left|\Lambda_{1}\right|^{-} /|z|}^{+\infty} \frac{1}{\sqrt{s}(s+1)} d s+\alpha \int_{\left|\Lambda_{1}\right|^{-} /|z|}^{+\infty} \frac{1}{s(s+1)} d s \\
& \leq p \pi \sqrt{|z|}+\alpha \log \left(1+\frac{|z|}{\left|\Lambda_{1}\right|}\right) .
\end{aligned}
$$

Since the function $z \in \mathbb{C} \longmapsto \alpha \log \left(1+|z| /\left|\Lambda_{1}\right|\right)-\sqrt{|z|}$ is bounded on $\mathbb{C}$, the lemma is proved.

Repeating the arguments, we obtain the same estimate for $f_{n}$.

Proof of Lemma A.4. To prove the result we are going to follow some ideas from [LK71] and [FR75] (see also [FCGBdT10]).

First, note that

$$
f^{\prime}\left(\Lambda_{n}\right)=-\frac{1}{\Lambda_{n}} \prod_{k \neq n}\left(1-\frac{\Lambda_{n}}{\Lambda_{k}}\right) \quad \forall n \geq 1 .
$$

Given $n \geq 1$, let us introduce the sets

$$
S_{1}(n)=\left\{k \neq n:\left|\Lambda_{k}\right| \leq 2\left|\Lambda_{n}\right|\right\} \quad \text { and } \quad S_{2}(n)=\left\{k:\left|\Lambda_{k}\right|>2\left|\Lambda_{n}\right|\right\}
$$

and the infinite product

$$
\mathcal{P}_{n}=\prod_{k \neq n}\left|1-\frac{\Lambda_{n}}{\Lambda_{k}}\right| .
$$

Let us give a lower bound for the product $\mathcal{P}_{n}$. To this end, we split this product into two parts using the sets $S_{1}(n)$ and $S_{2}(n)$.

1. From the definition of $S_{1}(n)$ and using 5 , we can write

$$
\begin{aligned}
\prod_{k \in S_{1}(n)}\left|1-\frac{\Lambda_{n}}{\Lambda_{k}}\right| & =\prod_{\substack{k \in S_{1}(n) \\
|k-n| \geq q}}\left|\frac{\Lambda_{k}-\Lambda_{n}}{\Lambda_{k}}\right| \prod_{\substack{k \in S_{1}(n) \\
|k-n|<q}}\left|\frac{\Lambda_{k}-\Lambda_{n}}{\Lambda_{k}}\right| \\
& \geq \prod_{\substack{k \in S_{1}(n) \\
|k-n| \geq q}} \frac{\rho}{2} \frac{|k-n|(k+n)}{\left|\Lambda_{n}\right|} \prod_{\substack{k \in S_{1}(n) \\
|k-n|<q}} \frac{1}{2} \frac{A}{\left|\Lambda_{n}\right|},
\end{aligned}
$$

where

$$
A=\inf _{k \neq n:|k-n|<q}\left|\Lambda_{k}-\Lambda_{n}\right|>0
$$

Copyright (c) by SIAM. Unauthorized reproduction of this article is prohibited. 
It follows that

$$
\prod_{k \in S_{1}(n)}\left|1-\frac{\Lambda_{n}}{\Lambda_{k}}\right| \geq \prod_{k \in S_{1}(n)} \frac{\rho}{2} \frac{|k-n|(k+n)}{\left|\Lambda_{n}\right|} \prod_{\substack{k \in S_{1}(n) \\|k-n|<q}} \frac{A}{\rho|k-n|(k+n)} .
$$

Since

$$
\prod_{\substack{k \in S_{1}(n) \\|k-n|<q}} \frac{A}{\rho|k-n|} \geq\left(\frac{A}{\rho q}\right)^{2 q-1} \prod_{\substack{k \in S_{1}(n) \\|k-n|<q}} \frac{1}{k+n} \geq \frac{1}{(2 n+q-1)^{2 q-1}}, \quad \forall n \geq 1,
$$

we deduce that

$$
\prod_{\substack{k \in S_{1}(n) \\|k-n|<q}} \frac{A}{|k-n|(k+n)} \geq \frac{C}{(2 n+q-1)^{2 q-1}} .
$$

As $\left|\Lambda_{n}\right| \geq C n^{2}$ for every $n \geq 1$ (see (A.1)), we obtain

$$
\prod_{\substack{k \in S_{1}(n) \\|k-n|<q}} \frac{A}{|k-n|(k+n)} \geq \frac{C}{\left|\Lambda_{n}\right|^{\frac{2 q-1}{2}}} .
$$

Let us define $r_{n}=\#\left\{k \in S_{1}(n): k<n\right\}$ and $s_{n}=\#\left\{k \in S_{1}(n): k>n\right\}$. From (A.1), we deduce that $k+n \geq C \sqrt{\left|\Lambda_{n}\right|}$ for any $n, k \geq 1$. Thus,

$$
\begin{aligned}
\prod_{k \in S_{1}(n)}\left|1-\frac{\Lambda_{n}}{\Lambda_{k}}\right| & \geq C\left|\Lambda_{n}\right|^{-q-\frac{1}{2}} r_{n} !\left(\frac{\rho \gamma_{2}}{2\left|\Lambda_{n}\right|^{1 / 2}}\right)^{r_{n}} s_{n} !\left(\frac{\rho \gamma_{2}}{2\left|\Lambda_{n}\right|^{1 / 2}}\right)^{s_{n}} \\
& =C\left|\Lambda_{n}\right|^{-q-\frac{1}{2}} \mathcal{P}_{n}^{(1)} \mathcal{P}_{n}^{(2)} \quad \forall n \geq 1 .
\end{aligned}
$$

Let us argue with $\mathcal{P}_{n}^{(1)}$. A similar reasoning will provide a lower bound for $\mathcal{P}_{n}^{(2)}$. Observe that there exist two constants $c_{0}, c_{1}>0$ such that

$$
r ! \geq c_{0}\left(\frac{r}{e}\right)^{r} \quad \forall r \geq 1
$$

and

$$
-c_{1}=\inf _{s>0} s(\log s)
$$

We can then write

$$
\begin{aligned}
\mathcal{P}_{n}^{(1)} & =r_{n} !\left(\frac{\rho \gamma_{2}}{2\left|\Lambda_{n}\right|^{1 / 2}}\right)^{r_{n}} \geq c_{0}\left(\frac{\rho \gamma_{2} r_{n}}{2 e\left|\Lambda_{n}\right|^{1 / 2}}\right)^{r_{n}} \\
& =c_{0} \exp \left[\frac{2 e\left|\Lambda_{n}\right|^{1 / 2}}{\rho \gamma_{2}}\left(\frac{\rho \gamma_{2} r_{n}}{2 e\left|\Lambda_{n}\right|^{1 / 2}}\right) \log \left(\frac{\rho \gamma_{2} r_{n}}{2 e\left|\Lambda_{n}\right|^{1 / 2}}\right)\right] \geq c_{0} \exp \left(-\frac{2 e c_{1}}{\rho \gamma_{2}}\left|\Lambda_{n}\right|^{1 / 2}\right) .
\end{aligned}
$$

Putting this inequality (and the similar one for the product $\mathcal{P}_{n}^{(2)}$ ) in (A.8) we obtain

$$
\prod_{k \in S_{1}(n)}\left|1-\frac{\Lambda_{n}}{\Lambda_{k}}\right| \geq e^{-C \sqrt{\left|\Lambda_{n}\right|}-C} \quad \forall n \geq 1
$$

Copyright $@$ ( ) by SIAM. Unauthorized reproduction of this article is prohibited. 
2. Let us now estimate the product (A.7) for $k \in S_{2}(n)$ which we denote by $\mathcal{P}_{n}^{(3)}$. Let $c_{2}>0$ be such that

$$
\log (1-s) \geq-c_{2} s \quad \forall s \in[0,1 / 2] .
$$

Observe that, for $k \in S_{2}(n)$ one has $\left|\Lambda_{n}\right| /\left|\Lambda_{k}\right| \leq 1 / 2$, so that we can use (A.10) to obtain

$$
\begin{aligned}
\log \mathcal{P}_{n}^{(3)} & \geq \sum_{k \in S_{2}(n)} \log \left(1-\frac{\left|\Lambda_{n}\right|}{\left|\Lambda_{k}\right|}\right) \geq-c_{2}\left|\Lambda_{n}\right| \sum_{k \in S_{2}(n)} \frac{1}{\left|\Lambda_{k}\right|}=-c_{2}\left|\Lambda_{n}\right| \int_{2\left|\Lambda_{n}\right|} \frac{1}{r} d \mathcal{N}(r) \\
& =-c_{2}\left|\Lambda_{n}\right|\left(-\frac{\mathcal{N}\left(2\left|\Lambda_{n}\right|\right)}{2\left|\Lambda_{n}\right|}+\int_{2\left|\Lambda_{n}\right|^{-}} \frac{\mathcal{N}(r)}{r^{2}} d r\right) \geq-c_{2}\left|\Lambda_{n}\right| \int_{2\left|\Lambda_{n}\right|^{-}} \frac{\mathcal{N}(r)}{r^{2}} d r \\
& \geq-c_{2}\left|\Lambda_{n}\right| \int_{2\left|\Lambda_{n}\right|^{-}} \frac{\alpha+p \sqrt{r}}{r^{2}} d r=-c_{2}\left|\Lambda_{n}\right|\left(\frac{\alpha}{2\left|\Lambda_{n}\right|}+\frac{2 p}{\sqrt{2\left|\Lambda_{n}\right|}}\right) \\
& =-\frac{\alpha c_{2}}{2}-\sqrt{2} p c_{2}\left|\Lambda_{n}\right|^{1 / 2}
\end{aligned}
$$

Putting (A.9) and this last inequality into (A.7), we deduce

$$
\mathcal{P}_{n}=\prod_{k \neq n}\left|1-\frac{\Lambda_{n}}{\Lambda_{k}}\right| \geq e^{-C \sqrt{\left|\Lambda_{n}\right|}-C} \quad \forall n \geq 1 .
$$

Since $\left|\Lambda_{n}\right| \geq\left|\Lambda_{1}\right|$ for every $n \geq 1$ (see assumption 4) we finally have

$$
\mathcal{P}_{n} \geq e^{-C \sqrt{\left|\Lambda_{n}\right|}} \quad \forall n \geq 1 .
$$

This inequality and formula (A.6) provide the desired estimate. This ends the proof.

Proof of Lemma A.5. For the proof we will follow some ideas from [FR71] and [Mil04] (see also [Red77]). Let us first consider again the counting function $N$ associated with the sequence $\left\{a_{n}\right\}_{n \geq 1}$ given by (4.7):

$$
N(r)=\#\left\{n \geq 1: a_{n} \leq r\right\}
$$

Observe again that the sequence $\left\{a_{n}\right\}_{n \geq 0}$ can be written as

$$
a_{n}=a_{0}+\frac{n^{2}}{A^{2}} \quad \forall n \geq 1 \quad \text { with } \quad A=\frac{d}{2} \quad \text { and } \quad a_{0}=\frac{d^{2}}{\tau^{2}}-\frac{4}{d^{2}}
$$

and that $a_{0}>0$ since we assumed that $\tau<d^{2} / 2$. Thus, $N(r)=0$ for $r<a_{1}$ and

$$
N(r)=\left\lfloor A \sqrt{r-a_{0}}\right\rfloor \quad \forall r \geq a_{1},
$$

We will often use that

$$
A \sqrt{r}-A \sqrt{a_{0}} \leq N(r) \leq A \sqrt{r} \quad \forall r \geq 0 .
$$

Let us prove the inequality (A.2). Observe that $M$ is an even function. So, we will show (A.2) for $x \in(0,+\infty)$. From the definition (4.8) of $M$, one has

$$
\log |M(x)|=\sum_{n \geq 1} \log \left|\frac{\sin \left(x / a_{n}\right)}{x / a_{n}}\right|=\int_{a_{1}^{-}}^{+\infty} g\left(\frac{x}{r}\right) d N(r),
$$

Copyright $($ C by SIAM. Unauthorized reproduction of this article is prohibited. 
where

$$
g(s)=\log \left|\frac{\sin s}{s}\right|, \quad s \in \mathbb{R} .
$$

- Since, $g$ is nonincreasing on $[0,1)$, for any $x \in\left[0, a_{1}\right]$ we have

$$
\log |M(x)| \leq \log |M(0)|=0 \leq-d \sqrt{x}+d \sqrt{a_{1}} \leq-d \sqrt{x}+\frac{d^{2}}{\tau},
$$

which gives the claim in that case.

- Assume now that $x>a_{1}$. We write

$$
\log |M(x)|=\sum_{a_{n} \leq x} g\left(x / a_{n}\right)+\sum_{a_{n}>x} g\left(x / a_{n}\right) \equiv I+J .
$$

Since $g$ is negative and nonincreasing on $[0,1]$, the second sum $J$ can be bounded as follows:

$$
\begin{aligned}
J & \leq \sum_{2 x \geq a_{n}>x} g\left(x / a_{n}\right) \leq-|g(1 / 2)|(N(2 x)-N(x)) \\
& \leq-|g(1 / 2)|\left(A \sqrt{2 x-a_{0}}-1-A \sqrt{x-a_{0}}\right) \\
& =|g(1 / 2)|-A|g(1 / 2)| \frac{x}{\sqrt{2 x-a_{0}}+\sqrt{x-a_{0}}} \\
& \leq|g(1 / 2)|-A \frac{|g(1 / 2)|}{\sqrt{2}+1} \sqrt{x} .
\end{aligned}
$$

In the first sum $I$, we use the inequality $g(s) \leq-\log s$ for any $s \geq 0$ to get

$$
\begin{aligned}
I & \leq-\sum_{a_{n} \leq x} \log \left(x / a_{n}\right)=\int_{a_{1}^{-}}^{x} \log \left(\frac{r}{x}\right) d N(r)=-\int_{a_{1}}^{x} \frac{N(r)}{r} d r \\
& \leq \int_{a_{1}}^{x} \frac{1-A \sqrt{r-a_{0}}}{r} d r \\
& =\log \left(x / a_{1}\right)-A\left(\int_{a_{1}}^{x} \frac{1}{\sqrt{r-a_{0}}} d r-a_{0} \int_{a_{1}}^{x} \frac{1}{r \sqrt{r-a_{0}}} d r\right) \\
& \leq \log \left(x / a_{1}\right)-2 A \sqrt{x-a_{0}}+2 A \sqrt{a_{1}-a_{0}}+A \sqrt{a_{0}} \int_{1}^{+\infty} \frac{1}{r \sqrt{r-1}} d r \\
& \leq-2 A \sqrt{x}+c_{1} A \sqrt{a_{0}}+\log (x)+2
\end{aligned}
$$

with $c_{1}=2+\int_{1}^{+\infty} \frac{1}{r \sqrt{r-1}} d r$.

Combining the two estimates gives

$$
\log |M(x)| \leq-A\left(2+\frac{|g(1 / 2)|}{1+\sqrt{2}}\right) \sqrt{x}+\log x+c_{1} A \sqrt{a_{0}}+2+|g(1 / 2)| .
$$

Observe now that $a_{0} \leq d^{2} / \tau^{2}$, that $2 A=d$, and that the function

$$
x \in\left[0,+\infty\left[\mapsto-A \frac{|g(1 / 2)|}{1+\sqrt{2}} \sqrt{x}+\log (x)+2+|g(1 / 2)|\right.\right.
$$

Copyright $@$ ( ) by SIAM. Unauthorized reproduction of this article is prohibited. 
is bounded by some number $c_{2}>0$ depending only on $A=d / 2$. We finally get the inequality

$$
\log |M(x)| \leq-d \sqrt{x}+\frac{c_{1} d^{2}}{2 \tau}+c_{2}
$$

which gives the claim by using that $1 \leq \frac{d^{2}}{2 \tau}$.

Proof of Lemma A.6. We start by observing that

$$
\frac{\sin i y}{i y}=\frac{\sinh y}{y} \geq 1 \quad \forall y \in \mathbb{R} .
$$

As a consequence, we obtain $M(i y) \geq 1$ for any $y \in \mathbb{R}$. Thus, we immediately get (A.3).

We will now obtain the proof of (A.4) by adapting the proof of Lemma 6.3 of [FR71] to the sequence $\left\{a_{n}\right\}_{n \geq 1}$ given by (4.7). We set $c_{0}=\log \sqrt{3}>0$.

- Assume first that $|y| / c_{0} \leq a_{1}$. Then, by using (A.3), we get

$$
\begin{aligned}
\log |M(i y)| & \geq 0 \geq \tau_{M}|y|-\tau_{M} c_{0} a_{1}=\tau_{M}|y|-\tau_{M} c_{0} \frac{d^{2}}{\tau^{2}} \\
& \geq \tau_{M}|y|-c_{0} \frac{d^{2}}{\tau} \quad \forall \frac{|y|}{c_{0}} \leq a_{1}=\frac{d^{2}}{\tau^{2}},
\end{aligned}
$$

and the claim is proved in that case.

- Assume now that $|y| / c_{0} \geq a_{1}$. Observe that

$$
\frac{\sin (i y)}{i y}=\frac{1}{2}\left(\frac{e^{y}-e^{-y}}{y}\right)=\frac{e^{|y|}-e^{-|y|}}{2|y|}=e^{|y|} \frac{1-e^{-2|y|}}{2|y|} \quad \forall y \neq 0 .
$$

Thus, using the definitions (4.8) and (4.9) of $M$ and $\tau_{M}$, we have

$$
\log |M(i y)|=\sum_{n \geq 1} \frac{|y|}{a_{n}}+\sum_{n \geq 1} \log \left(\frac{1-e^{-2|y| / a_{n}}}{2|y| / a_{n}}\right)=\tau_{M}|y|+\mathcal{I}
$$

where the sequence $\left\{a_{n}\right\}_{n \geq 1}$ is given by (4.7).

In order to bound the series $\mathcal{I}$, we will use the inequalities

$$
\frac{1-e^{-2 y}}{2 y} \geq e^{-2 y} \quad \forall y>0 \quad \text { and } \quad \frac{1-e^{-2 y}}{2 y} \geq \frac{1}{3 y} \quad \forall y \geq \log \sqrt{3}=c_{0} .
$$

So, for $y \in \mathbb{R}$ with $|y| / c_{0} \geq a_{1}$, one has

$$
\mathcal{I}=\sum_{n \geq 1} \log \left(\frac{1-e^{-2|y| / a_{n}}}{2|y| / a_{n}}\right) \geq-\sum_{\substack{n \geq 1 \\ a_{n}>|y| / c_{0}}} \frac{2|y|}{a_{n}}+\sum_{\substack{n \geq 1 \\ a_{n} \leq|y| / c_{0}}} \log \left(\frac{a_{n}}{3|y|}\right) \equiv \mathcal{I}_{1}+\mathcal{I}_{2} .
$$

- Let us first bound from below $\mathcal{I}_{1}$ in the expression (A.14). One has

$$
\mathcal{I}_{1}=-\sum_{\substack{n \geq 1 \\ a_{n}>|y| / c_{0}}} \frac{2|y|}{a_{n}}=-2|y| \int_{a_{n_{0}}^{-}}^{+\infty} \frac{d N(r)}{r} \geq-2|y| \int_{|y| / c_{0}}^{+\infty} \frac{d N(r)}{r},
$$

Copyright (c) by SIAM. Unauthorized reproduction of this article is prohibited. 
where $n_{0} \geq 1$ is the smallest integer such that $a_{n_{0}}>|y| / c_{0}$ and $N(\cdot)$ is the counting function associated to the sequence $\left\{a_{n}\right\}_{n \geq 1}$ (see (A.11) and (A.12)). Integrating by parts, we obtain

$$
\begin{aligned}
\mathcal{I}_{1} & \geq-2|y|\left[\left.\frac{1}{r} N(r)\right|_{|y| / c_{0}} ^{+\infty}+\int_{|y| / c_{0}}^{+\infty} \frac{N(r)}{r^{2}} d r\right] \\
& \geq-2|y| A \int_{|y| / c_{0}}^{+\infty} \frac{\sqrt{r-a_{0}}}{r^{2}} d r \\
& \geq-2 A|y| \int_{|y| / c_{0}}^{+\infty} r^{-3 / 2} d r
\end{aligned}
$$

that is to say,

$$
\mathcal{I}_{1} \geq-4 c_{0}^{1 / 2} A \sqrt{|y|} \quad \forall \frac{|y|}{c_{0}}>a_{1}
$$

- Let us deal with the second term $\mathcal{I}_{2}$ in (A.14) for $|y|$ satisfying $a_{1}<$ $|y| / c_{0}$. Using that for any $r \in\left[a_{1},|y| / c_{0}\right]$ one has $r<3|y|\left(c_{0}=\log \sqrt{3}\right)$, we can write

$$
\begin{aligned}
\mathcal{I}_{2} & =\sum_{\substack{n \geq 1 \\
a_{n} \leq|y| / c_{0}}} \log \left(\frac{a_{n}}{3|y|}\right)=\int_{a_{1}^{-}}^{a_{n_{1}}} \log \left(\frac{r}{3|y|}\right) d N(r) \\
& \geq \int_{a_{1}^{-}}^{|y| / c_{0}} \log \left(\frac{r}{3|y|}\right) d N(r),
\end{aligned}
$$

where $n_{1} \geq 1$ is the largest integer such that $a_{n_{1}} \leq|y| / c_{0}$. Again, integrating by parts, we deduce

$$
\begin{aligned}
\mathcal{I}_{2} & \geq\left. N(r) \log \left(\frac{r}{3|y|}\right)\right|_{a_{1}^{-}} ^{|y| / c_{0}}-\int_{a_{1}^{-}}^{|y| / c_{0}} \frac{N(r)}{r} d r \\
& =-\log \left(3 c_{0}\right) N\left(|y| / c_{0}\right)-A \int_{a_{1}^{-}}^{|y| / c_{0}} \frac{\sqrt{r-a_{0}}}{r} d r \\
& \geq-\log \left(3 c_{0}\right) N\left(|y| / c_{0}\right)-A \int_{0}^{|y| / c_{0}} \frac{1}{\sqrt{r}} d r \\
& \geq-A\left(2+\log \left(3 c_{0}\right)\right) c_{0}^{-1 / 2} \sqrt{|y|} .
\end{aligned}
$$

In view of (A.13) and (A.14), this last inequality together with (A.15) provides $\left(c_{0}=\log \sqrt{3}\right)$

$$
\log |M(i y)| \geq \tau_{M}|y|-c_{1} d \sqrt{|y|}
$$

with $c_{1}=\left(1+2 c_{0}+\log \left(3 c_{0}\right) / 2\right) c_{0}^{-1 / 2}$.

Owing to the previous calculations, we finally obtain the inequality (A.4). This ends the proof.

Copyright $@$ by SIAM. Unauthorized reproduction of this article is prohibited. 


\section{REFERENCES}

$[\mathrm{AB} 12]$

[ABL12]

[AKBDGB09a]

[AKBDGB09b]

[AKBGBdT11a]

[AKBGBdT11b]

[AKBGBdT13a]

[AKBGBdT13b]

[BDR07]

[BN02]

[DR77]

[Fat75]

[FCGBdT10]

[FCZ00]

[FR71]

[FR75]

[Güi85]

[KFA69]

[LK71]

[LR07]
F. Alabau-Boussouira, Controllability of cascade coupled systems of multidimensional evolution PDEs by a reduced number of controls, C. R. Acad. Sci. Paris Sér. I Math., 350 (2012), pp. 577-582.

F. Alabau-Boussouira AND M. LÉAUtAud, Indirect stabilization of locally coupled wave-type systems, ESAIM Control Optim. Calc. Var., 18 (2012), pp. 548-582.

F. Ammar-Khodja, A. Benabdallah, C. Dupaix, and M. González-Burgos, A generalization of the Kalman rank condition for time-dependent coupled linear parabolic systems, Differential Equations Appl., 1 (2009), pp. 139151.

F. Ammar-Khodja, A. Benabdallah, C. Dupaix, and M. González-Burgos, A Kalman rank condition for the localized distributed controllability of a class of linear parabolic systems, J. Evol. Equations, 9 (2009), pp. 267-291.

F. Ammar-Khodja, A. Benabdallah, M. González-Burgos, and L. DE TERESA, The Kalman condition for the boundary controllability of coupled parabolic systems. Bounds on biorthogonal families to complex matrix exponentials, J. Math. Pures Appl. (9), 96 (2011), pp. 555-590.

F. Ammar-Khodja, A. Benabdallah, M. GonzÁlez-Burgos, and L. DE TERESA, Recent results on the controllability of linear coupled parabolic problems: A survey, Math. Control Related Fields, 1 (2011), pp. 267-306.

F. Ammar-Khodja, A. Benabdallah, M. González-Burgos, and L. DE TEReSA, A new relation between the condensation index of complex sequences and the null controllability of parabolic systems, C. R. Acad. Sci. Paris Sér. I Math., 351 (2013), pp. 743-746.

F. Ammar-Khodja, A. Benabdallah, M. González-Burgos, and L. DE TERESA, Minimal time for the null controllability of parabolic systems: The effect of the condensation index of complex sequences, J. Funct. Anal., 267 (2014), pp. 2077-2151.

A. Benabdallah, Y. Dermenjian, and J. Le Rousseau, On the controllability of linear parabolic equations with an arbitrary control location for stratified media, C. R. Acad. Sci. Paris Sér. I Math., 344 (2007), pp. 357-362.

A. Benabdallah And M. G. NASO, Null controllability of a thermoelastic plate, Abstr. Appl. Anal., 7 (2002), pp. 585-599.

S. Dolecki And D. L. Russell, A general theory of observation and control, SIAM J. Control Optim., 15 (1977), pp. 185-220.

H. O. FATTORIni, Boundary control of temperature distributions in a parallelepipedon, SIAM J. Control, 13 (1975), pp. 1-13.

E. Fernández-Cara, M. González-Burgos, and L. de Teresa, Boundary controllability of parabolic coupled equations, J. Funct. Anal., 259 (2010), pp. $1720-1758$.

E. Fernández-CARA And E. ZuazuA, The cost of approximate controllability for heat equations: The linear case, Adv. Differential Equations, 5 (2000), pp. $465-514$.

H. O. FAtTorini And D. L. Russell, Exact controllability theorems for linear parabolic equations in one space dimension, Arch. Ration. Mech. Anal., 43 (1971), pp. 272-292.

H. O. FATtORIni AND D. L. Russell, Uniform bounds on biorthogonal functions for real exponentials with an application to the control theory of parabolic equations, Quart. Appl. Math., 32 (1974), pp. 45-69.

E. N. Güichal, A lower bound of the norm of the control operator for the heat equation, J. Math. Anal. Appl., 110 (1985), pp. 519-527.

R. E. Kalman, P. L. Falb, And M. A. ArbiB, Topics in Mathematical Control Theory, McGraw-Hill, New York, 1969.

W. A. J. Luxemburg And J. KorevaAr, Entire functions and Müntz-Szász type approximation, Trans. Amer. Math. Soc., 157 (1971), pp. 23-37.

J. Le Rousseau, Représentation Microlocale de Solutions de Systèmes Hyperboliques, Application à l'Imagerie, et Contributions au Contrôle et aux Problèmes Inverses pour des Equations Paraboliques, Habilitation thesis, Aix-Marseille Université, http://hal.archive-souvertes.fr/tel-00201887/fr/ (2007). 
[LR95] G. Lebeau And L. Robbiano, Contrôle exact de l'équation de la chaleur, Comm. Partial Differential Equations, 20 (1995), pp. 335-356.

[LZ98] G. Lebeau And E. Zuazua, Null-controllability of a system of linear thermoelasticity, Arch. Ration. Mech. Anal., 141 (1998), pp. 297-329.

[Mil04] L. Miller, Geometric bounds on the growth rate of null-controllability cost for the heat equation in small time, J. Differential Equations, 204 (2004), pp. 202-226.

[Mil05] L. Miller, On the null-controllability of the heat equation in unbounded domains, Bull. Sci. Math., 129 (2005), pp. 175-185.

[Oli13] G. OLIVE, Boundary approximate controllability of some linear parabolic systems, Evol. Equations Control Theory, 3 (2014), pp. 167-189.

[Red77] R. M. RedhefFer, Completeness of sets of complex exponentials, Adv. Math., 24 (1977), pp. 1-62.

[Rud74] W. Rudin, Real and Complex Analysis, 2nd ed., McGraw-Hill, New York, 1974.

[Sei84] T. I. SEIDman, Two results on exact boundary control of parabolic equations, Appl Math Optim., 11 (1984), pp. 145-152.

[TT11]

[dT00] L. DE TERESA, Insensitizing controls for a semilinear heat equation, Comm. Partial Differential Equations, 25 (2000), pp. 39-72.

Copyright (c) by SIAM. Unauthorized reproduction of this article is prohibited. 